The Astrophysical Journal, 485:533-551, 1997 August 20

(C) 1997. The American Astronomical Society. All rights reserved. Printed in U.S.A.

\title{
DYNAMICS AND STRUCTURE OF THREE-DIMENSIONAL POLOIDALLY MAGNETIZED SUPERMAGNETOSONIC JETS
}

\author{
PHILIP E. HARDEE \\ Department of Physics and Astronomy, The University of Alabama, Tuscaloosa, AL 35487; hardee@venus.astr.ua.edu \\ David A. ClaRke \\ Department of Astronomy and Physics, Saint Mary's University, Halifax, Nova Scotia, Canada B3H 3C3; dclarke@ap.stmarys.ca \\ AND \\ AleXANDER Rosen \\ Department of Physics and Astronomy, The University of Alabama, Tuscaloosa, AL 35487; rosen@eclipse.astr.ua.edu \\ Received 1996 October 23; accepted 1997 March 24
}

\begin{abstract}
A set of three-dimensional magnetohydrodynamical simulations of supermagnetosonic magnetized jets has been performed. The jets contain an equipartition primarily poloidal magnetic field, and the effect of jet density on jet dynamics and structure is evaluated. The jet is precessed at the origin to break the symmetry and to excite Kelvin-Helmholtz-unstable helical modes. In the linear limit, observed structures are similar in all simulations and can be produced by structures predicted to arise as a result of instability. The amplitude of various unstable modes is evaluated. Most unstable modes do not reach the maximum amplitudes estimated from the linear theory by computing displacement surfaces associated with the modes. Surprisingly, even these large-amplitude distortions are fitted reasonably well by displacement surfaces computed from the linear theory. Large-amplitude helical and elliptical distortions lead to significant differences in the nonlinear development of the jets as a function of the jet density. Jets less dense than the surrounding medium entrain material, lose energy through shock heating, and slow down relatively rapidly once large-amplitude distortions develop as a result of instability. Jets more dense than the surrounding medium lose much less energy as they entrain and accelerate the surrounding medium. The dense jet maintains a high-speed spine that exhibits large-amplitude helical twisting and elliptical distortion over considerable distance without disruption of internal jet structures as happens for the less dense jets. This dense high-speed spine is surrounded by a less dense sheath consisting of slower moving jet fluid and magnetic field mixed with the external medium. Simulated synchrotron intensity and fractional polarization images from these calculations provide a considerably improved connection between simulation results and jet observations than do images made using the fluid variables alone. Intensity structure in the dense jet simulation appears remarkably similar to structure observed in the Cygnus A jet. These simulations suggest that the extended jets in high-power radio sources propagate to such large distances without disruption by entrainment because they are surrounded by a lobe or cocoon whose density is less than the jet density.
\end{abstract}

Subject headings: galaxies: jets — hydrodynamics — instabilities - MHD

\section{INTRODUCTION}

The highly collimated outflows from extragalactic radio sources and protostellar systems, e.g., HH 34 (Burke, Mundt, \& Ray 1988), and probably also the Galactic superluminals GRS 1915+ 105 (Mirabel \& Rodríguez 1994) and GRO J1655-40 (Tingay et al. 1995; Hjellming \& Rupen 1995 ) are subject to instability that can produce organized helical structures and will lead to momentum and mass exchange with the external environment. Observation of extragalactic jets shows that jets can propagate many tens of times their diameter while remaining highly collimated, e.g., Cygnus A (Dreher, Carilli, \& Perley 1987) and NGC 6251 (Perley, Bridle, \& Willis 1984) or lose their narrow collimation after a relatively short distance, e.g., M84 (Laing $\&$ Bridle 1987). Observation also shows that jets can show bulk large-scale sinusoidal distortion, e.g., 3C 449 (Perley \& Cornwell 1984) or exhibit smaller scale apparent sinusoidal oscillations, e.g., HH 34 and GRO J1655-40, which, if the jets are not ballistic, may be related to flow instability possibly triggered by precession or orbital motion associated with the central engine. In this case, it is hoped that observed jet structures can be related to jet velocity, density, and magnetic fields via numerical simulations, stability theory, and modeling. This is of particular importance to extragalactic jets or those Galactic jets in which the continuum nature of synchrotron and inverse Compton emission provides no direct measure of these quantities and whose apparent motions may reflect a wave pattern or shock speed different from the speed of the underlying flow.

Theoretical results and laboratory experiments (see Bicknell 1984, 1986a, 1986b) and three-dimensional numerical simulations (Hardee, Clarke, \& Howell 1995) have indicated that momentum and mass exchange with an external environment more dense than a fluid jet results in a relatively rapid loss of the initial high collimation, and the outward flow broadens and slows as denser external material is mixed with and accelerated by the lighter jet fluid. On the other hand, extragalactic jets in FR II-type radio sources that remain highly collimated are those surrounded by a lobe or cocoon almost certainly less dense than the jet (Norman, Winkler, \& Smarr 1984). In addition, the Galactic protostellar jets are perhaps 10 times more dense than the immediately surrounding medium. It is also possible that the inclusion of magnetic fields reduces 
momentum and mass exchange with the external environment. That a sufficiently supermagnetosonic magnetized jet denser than the immediately surrounding medium might mitigate the Kelvin-Helmholtz disruption of extragalactic jets was suggested by a three-dimensional numerical simulation performed by Hardee \& Clarke (1995). In this paper, we report on results obtained from three numerical simulations of magnetized cylindrical jets that are initialized across a Cartesian grid. Thus, these simulations are relevant to astrophysical jets far behind the propagating jet working surface. The principal difference between the simulations is the different jet density. The inclusion of magnetic fields in these simulations allows an examination of the dynamical effects that magnetic fields have on stability through comparison with previous purely fluid jet simulations. The magnetic fields also allow computation of total intensity and fractional polarization images that provide a better connection to the observations than do the fluid variables.

\section{NUMERICAL SIMULATIONS}

Simulations were performed using the three-dimensional MHD code ZEUS-3D, an Eulerian finite-difference code using the recently developed consistent method of characteristics $(\mathrm{CMoC})$. The strength of the $\mathrm{CMoC}$ lies in its ability to solve the transverse momentum transport and magnetic induction equations simultaneously and in a planar split fashion. Additional details may be found in Clarke (1996). The development of CMoC has allowed us to perform three-dimensional MHD calculations such as those presented here with some confidence. Interpolations are carried out by a second-order-accurate monotonic upwinded time-centered scheme (van Leer 1977), and a von Neumann-Richtmyer artificial viscosity is used to stabilize shocks. The MHD test suites as described by Stone et al. (1992) and Clarke (1996) were used to establish the reliability of the techniques.

\subsection{Initialization}

Simulations are initialized by establishing a cylindrical jet across a three-dimensional Cartesian grid resolved into $130 \times 130 \times 325$ zones. Thirty uniform zones span the jet diameter along the transverse Cartesian axes ( $y$-axis and $z$-axis). Outside the jet, the grid zones are ratioed where each subsequent zone increases in size by a factor 1.05 . Altogether, the 130 zones along the transverse Cartesian axes span a total distance of 30 jet radii. Along the $x$-axis, 225 uniform zones span a distance of 30 jet radii outward from the jet origin. An additional 100 ratioed zones span an additional distance of 30 jet radii, where each subsequent zone increases in size by a factor 1.0148 . Outflow boundary conditions are used at all boundaries except where the jet enters the grid where inflow boundary conditions are used. The use of a nonuniform grid such as we are employing here has been shown to have the beneficial effect of reducing reflections off the grid boundaries as a result of increased dissipation of disturbances by the larger grid zones near the grid boundaries (Bodo et al. 1995). With 15 zones across the jet radius in the transverse direction and 7.5 zones per jet radius in the axial direction, we should be able to detect structures with length scales larger than about $25 \%$ of the jet radius in the transverse direction and $50 \%$ of the jet radius in the axial direction.

The "equilibrium jets" (as opposed to "propagating jets") shown in $\S \S 2.2-2.4$ are initialized with a uniform density $\rho_{\mathrm{jt}}$ across the computational grid within an external medium of uniform density $\rho_{\mathrm{ex}}$. The thermal pressure in the jet is initialized at $p_{\mathrm{jt}}^{\mathrm{th}}=0.54 p_{\mathrm{ex}}$, where $p_{\mathrm{ex}}$ is the pressure in the uniform external medium. The magnetic field in the jet is initialized with a uniform poloidal component, $B_{x}$, corresponding to a magnetic pressure $p_{\mathrm{jt}}^{B_{x}}=0.469 p_{\mathrm{ex}}$. In the absence of a toroidal magnetic component, this leads to a small $(\sim 1 \%)$ overpressure of the jet compared to the external medium. However, a toroidal magnetic component is included in the jet, and its variation with radius is shown in Figure 1. The toroidal component has a maximum value of $B_{\phi}^{\mathrm{pk}} \simeq 0.339 B_{x}$, gives a helical twist to the jet magnetic field, and provides a confining magnetic pressure which at maximum value is equal to $\sim 11.6 \%$ of the poloidal magnetic pressure. The total transverse static jet pressure varies from greater than the external pressure by about $1 \%$ at jet center and near to the jet surface to about $5 \%$ less than the external pressure where the toroidal magnetic component is a maximum. The helical pitch of the magnetic field is also indicated in Figure 1. The maximum pitch of $\sim 18^{\circ} .7$ at a radial distance of $0.8 R$ corresponds to a $360^{\circ}$ twist around the jet in an axial distance of $\sim 14.8 R$. This primarily poloidal magnetic field configuration of the three simulations shown in $\S \S 2.2-2.4$ has been chosen so that structures arising in the numerical simulations can be directly compared to structures predicted by linear stability theory $(\$ \S 3$ and 4). Additional simulations with different magnetic field configurations and strengths have also been performed, but a detailed analysis of the results of these additional simulations is beyond the scope of this paper.

The initial conditions for the three simulations are given in Table 1. In all simulations, the external density and temperature are the same. The jet density is varied, and the jet temperature is varied appropriately to maintain a constant jet thermal pressure. The internal sonic, Alfvénic, and magnetosonic Mach numbers are defined as $M_{\mathrm{jt}} \equiv u / a_{\mathrm{jt}}, M_{\mathrm{jt}}^{\mathrm{A}} \equiv$ $u / V_{\mathrm{A}, \mathrm{jt}}$, and $M_{\mathrm{jt}}^{\mathrm{ms}} \equiv u /\left(a_{\mathrm{jt}}^{2}+V_{\mathrm{A}, \mathrm{jt}}^{2}\right)^{1 / 2}$, respectively, where $a_{\mathrm{jt}}$ and $V_{\mathrm{A}, \mathrm{jt}}$ are the jet sound speed and the poloidal Alfvén speed, respectively, and we define a jet magnetosonic speed as $a_{\mathrm{jt}}^{\mathrm{ms}} \equiv\left(a_{\mathrm{jt}}^{2}+V_{\mathrm{A}, \mathrm{ji}}^{2}\right)^{1 / 2}$. The jet speed is initialized to keep the jet magnetosonic Mach number within a relatively small range. For sufficiently supermagnetosonic jets such as we study here, the theoretically predicted stability behavior is

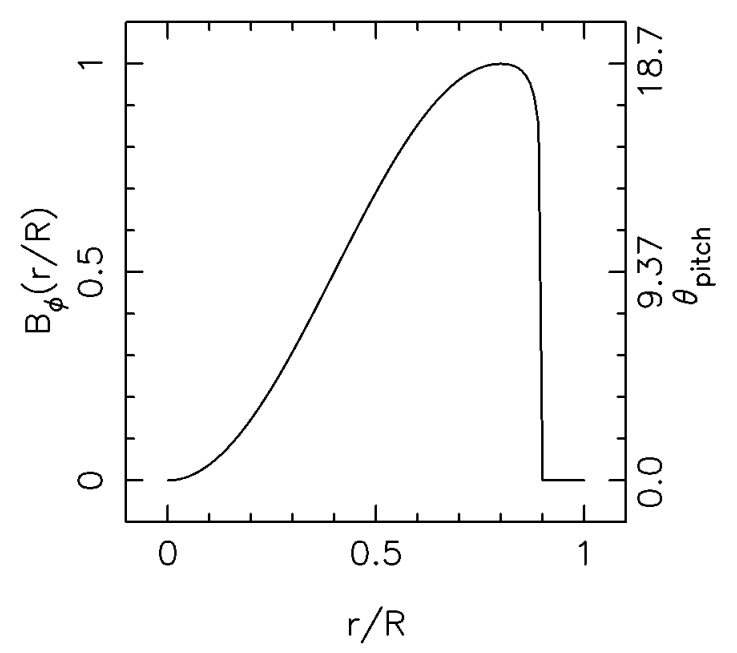

FIG. 1.-Toroidal component and helical pitch of the magnetic field as a function of the jet radius. 
TABLE 1

INITIAL CONDITIONS

\begin{tabular}{lcccccccc}
\hline \hline Simulation & $\eta$ & $a_{\mathrm{jt}} / a_{\mathrm{ex}}$ & $V_{\mathrm{A}, \mathrm{jt}} / a_{\mathrm{ex}}$ & $a_{\mathrm{jt}}^{\mathrm{ms}} / a_{\mathrm{ex}}$ & $M_{\mathrm{ex}}$ & $M_{\mathrm{jt}}$ & $M_{\mathrm{jt}}^{\mathrm{A}}$ & $M_{\mathrm{jt}}^{\mathrm{ms}}$ \\
\hline A $\ldots \ldots \ldots \ldots$ & 0.25 & 1.47 & 1.50 & 2.10 & 12.50 & 8.50 & 8.33 & 5.95 \\
$\mathrm{~B} \ldots \ldots \ldots$ & 1.00 & 0.74 & 0.75 & 1.05 & 5.14 & 7.00 & 6.86 & 4.90 \\
$\mathrm{C} \ldots \ldots \ldots$ & 4.00 & 0.37 & 0.38 & 0.52 & 4.04 & 11.0 & 10.78 & 7.70 \\
\hline
\end{tabular}

primarily a function of the jet magnetosonic Mach number. Differences between the simulations are expected to be the result of nonlinear behavior primarily related to the density difference and secondarily related to the velocity difference. Some difference between the present and previous purely fluid simulations (Hardee et al. 1995) may be related to the addition of the relatively strong equipartition magnetic field. With jet speeds corresponding to magnetosonic Mach numbers, $M_{\mathrm{jt}}^{\mathrm{ms}}$, of $(a) \sim 5.95$, (b) $\sim 4.90$, and $(c) \sim 7.70$, one flow through time (i.e., the time required for jet material to flow completely across the computational grid) is $\tau_{f} \equiv$ $t_{f} a_{\mathrm{ex}} / R \simeq(a) 4.8,(b) 11.6$, and (c) 14.8, where $a_{\mathrm{ex}}$ is the sound speed in the external medium.

In the simulations presented here, the cylindrical jet is driven by a periodic precession of the jet velocity, $u$, at the inlet with an angle of 0.005 radian relative to the $x$-axis in a counterclockwise sense when viewed outward from the inlet and with an angular frequency $(a$ and $b) \omega=1.6 a_{\mathrm{ex}} / R$, and (c) $\omega=1.2 a_{\mathrm{ex}} / R$. These precessional frequencies are near the theoretically predicted maximally unstable frequency associated with helical twisting of the jet and serve to excite other Kelvin-Helmholtz-unstable helical modes. With these precession frequencies, one complete precessional period occurs in a time ( $a$ and $b) \tau_{p} \equiv t_{p} a_{\mathrm{ex}} / R \simeq 3.93$, and $(c) \tau_{p} \simeq$ 5.24. The initial transverse motion imparted to the jet by the precession in the simulations is $(a) \simeq 0.030 a_{\mathrm{jt}}^{\mathrm{ms}}$ or $\simeq 0.062 a_{\mathrm{ex}}$, (b) $\simeq 0.025 a_{\mathrm{jt}}^{\mathrm{ms}}$ or $\simeq 0.026 a_{\mathrm{ex}}$, and $(c) \simeq 0.039 a_{\mathrm{jt}}^{\mathrm{ms}}$ or $\simeq 0.020 a_{\text {ex }}$ and is well within the linear regime of a small perturbation. The direction of precession induces a helical twist in the same sense as that of the magnetic field helicity so that the toroidal component of the field has relatively little influence on the development of unstable growing waves, i.e., helical wavefronts are at shallow angles to the helically twisted magnetic field (Hardee et al. 1992a), and this choice also guarantees that the magnetic field experiences the maximum compressive effects associated with the helical perturbations induced by the precession. After dynamical times $\tau_{d} \equiv t_{d} a_{\mathrm{ex}} / R=(a) 17$, (b) 28 , and (c) 36 , the numerical simulations have reached a quasi-steady state out to axial distances in excess of $50 R$. These dynamical times correspond to more than four precession periods and about three flow-through times through an axial distance of $50 R$.

Images from the simulations consist of magnetic pressure cross sections and line-of-sight integrations corresponding to total synchrotron intensity and fractional polarization. The cross section images of dimension $10 R \times 10 R$ are oriented with the $y$-axis and $z$-axis in the vertical and horizontal directions, respectively, and with the $x$-axis into the page, i.e., we look outward along the flow direction. To generate intensity and fractional polarization images, an emissivity is defined by $p^{\text {th }}(B \sin \theta)^{3 / 2}$, where $\theta$ is the angle made by the local magnetic field with respect to the line of sight. This emissivity can be shown to mimic synchrotron emission from a system in which the energy and number densities of the relativistic particles are proportional to the energy and number densities of the thermal fluid. This simplistic assumption is necessary when the relativistic particles are not explicitly tracked (Clarke, Norman, \& Burns 1989). The Stokes parameters are computed from line-ofsight integrations along the $z$-axis, and from these parameters, the simulated intensity and fractional polarization images are generated. The displayed images have a dimension of $20 R \times 60 R$. Recall that the computational grid has dimension $30 R \times 30 R \times 60 R$. We note that an image formed by a line-of-sight integration of the magnetic pressure is very similar in appearance to the total intensity image. In addition, the magnetic pressure cross sections and line-of-sight images reveal the extent of jet spreading and structure as only the jet fluid is magnetized.

\subsection{Magnetic Pressure Cross Sections and Line-of-Sight Images}

Figures 2, 3, 4, and 5 (Plates 9-12) each contain eight magnetic pressure cross section images, at axial distances from $6 R$ to $48 R$ in increments of $6 R$, and two line-of-sight images. Figures 2 and 3 are from simulations A (light jet) and $B$ (equal density jet), respectively. Figures 4 and 5 are from simulation $\mathrm{C}$ (heavy jet) at dynamical times $\tau_{d}=34$, and $\tau_{d}=36$, respectively. In simulation A (Fig. 2), the images show that out to axial distances of $\sim 24 R$, the jet remains well collimated and shows evidence for organized internal jet structure. Fluting of the jet surface is apparent in the cross section at $12 R$, and the cross sections show some jet flattening with a twist through $\sim 180^{\circ}$ between $12 R$ and $24 R$. The line-of-sight intensity image shows twisted bright filaments that are related to fluting and flattening of the jet. In simulation B (Fig. 3), the jet remains well collimated and shows evidence for organized internal jet structure out to axial distances of $\sim 29 R$. Surface fluting is very pronounced in the cross section at $12 R$ (seven ripples are apparent), and the cross sections show evidence for flattening particularly at an axial distance of $30 R$. Cross sections at other dynamical times suggest a flattening that twists through $180^{\circ}$ over a length scale of $\sim 13.5 R$. At axial distances between $16 R$ and $25 R$, dual filamentation twists through $180^{\circ}$ over a length scale of $\sim 4.5 R$ (see Fig. 8 in Hardee 1995).

In simulation $C$ (Figs. 4 and 5), the images suggest that the flow remains relatively highly collimated across the entire computational grid. For details of the structures seen at $\tau_{d}=34$, refer to Hardee \& Clarke (1995). Small-scale surface fluting like that seen in the less dense jets is not apparent in these images, but instead the jet appears square in cross sections out to $\sim 24 R$. Animation of the cross sections reveals that the square distortion is only partially an effect of the Cartesian grid and that the distortion rotates temporally and spatially. Interestingly, the square distortion does not show a smooth rotation in temporal animations but rather shows a square pattern that changes temporally in orientation in $45^{\circ}$ increments after passing 
through a fluted cross section stage. The lack of change in orientation between the panels from $6 R$ to $24 R$ and between the two figures is the serendipitous result of a $90^{\circ}$ spatial rotation with wavelength $\approx 6 R$ (the spatial separation between the panels) and a $90^{\circ}$ temporal rotation which occurs in $\Delta \tau_{d} \approx 2$ (the temporal separation between the figures). In $\S 4$, we will see that an additional triangular distortion (suggested by the cross section at $18 R$ in Fig. 4) with a $120^{\circ}$ spatial rotation in a wavelength $\approx 9 R$ is also required at these axial distances.

A small-amplitude sinusoidal oscillation in the line-ofsight images (helical twist of the jet) is evident in simulations $\mathrm{A}$ and $\mathrm{B}$ at axial distances $<30 R$ with wavelengths $\approx 20 R$ and $\approx 11.5 R$, respectively. The helical twist moves outward with a wave speed $v_{w} \approx 0.30 u$ and $v_{w} \approx 0.54 u$ in simulations $A$ and $B$, respectively. At larger axial distances in simulation $\mathrm{A}$, there is evidence for a helical twist but with wavelength decreasing to $\sim 16 R$. In simulation $\mathrm{B}$, at times earlier than shown in the present image, we find evidence for sinusoidal oscillation with a wavelength $\approx 5.5 R$ at axial distances $15 R-30 R$ that are not readily identifiable with features in the cross sections. Simulation C exhibits a relatively well defined large-scale sinusoidal oscillation with a wavelength $\approx 14 R$ across most of the computational grid. The oscillation moves outward by $\sim 6 R$ between the two images. This motion corresponds to a wave speed of $v_{w} \approx 0.74 u$.

All jets become less well collimated in the outer half of the computational grid. In simulation A, beyond about $27 R$, organized structure is disrupted, and jet material can be found up to a distance of $\sim 5 R$ from the jet axis at large axial distances. For example, magnetized jet material fills much of the $10 R \times 10 R$ cross section at an axial distance of $48 R$ and is clearly mixed with unmagnetized external material. In simulation $\mathrm{B}$, the flow begins to broaden significantly at an axial distance of about $30 R$, and beyond this distance organized structure is much less well defined. While magnetized jet material can be found up to a distance of $\sim 5 R$ from the jet axis and magnetized jet material fills a considerable portion of the $10 R \times 10 R$ cross section at an axial distance of $48 R$, there is more central concentration than in simulation A. There is a suggestion of dual magnetic filaments in the cross section at $48 R$ in simulation B. In simulation $C$, magnetized jet material can be found only up to a distance of $\sim 2.5 R$ in the cross section images, and this reflects the strong central concentration of the jet although in this case not the true extent of mixing.

In simulation $\mathrm{C}$, organized internal structure and collimation beyond an axial distance of $30 R$ remain even after a helical twist and jet flattening have grown to significant amplitudes. Jet flattening appears in the line-of-sight images as alternating broad and narrow regions that move outward with a wave speed similar to the wave speed of the large-scale sinusoidal oscillation. The cross section images suggest that magnetic filaments lie at the ends of the long axis of the flattened jet. At a fixed distance, the flattened jet rotates counterclockwise by almost $90^{\circ}$ between Figures 4 and 5 and rotates in the same sense as jet precession at the origin. We conclude that the jet is subject to flattening and dual filamentation that twists through $180^{\circ}$ with a wavelength $\lambda / R \approx 14$. The flattening goes through a nearly $360^{\circ}$ rotation without disruption. Note that the $360^{\circ}$ helical twist induced by precession at the jet origin occurs over approximately the same axial distance as the $180^{\circ}$ twist in jet flattening. There is also some evidence of filament twisting with wavelengths $\approx 4 R$ inside the jet. Additional complex structures on relatively short scale lengths are also revealed in the intensity images at distances between $12 R$ and $24 R$ and appear to be associated with structures helically twisted around the jet both at the jet surface and in the jet interior but are not easily related to features in the cross sections.

In simulation $\mathrm{C}$, the bright diagonal structures crossing the jet from left bottom to right top in the total intensity images appear to correspond to those locations where magnetic filaments associated with jet flattenting are aligned with the leading edge of the jet helicity induced by precession at the origin. Note the line-of-sight merging of the twin diagonal features in the intensity image in Figure 4 at axial distances of $\sim 24 R$ and $\sim 38 R$ into single diagonal features at axial distances of $\sim 32 R$ and $\sim 44 R$ in the intensity image in Figure 5. This change implies that different structures, e.g., helical twisting and jet flattening, do not move with exactly the same speed. Note that other small scale cross section and intensity features change significantly without simple relation between Figures 4 and 5 .

In all simulations out to $\sim 15 R$, the fractional polarization is higher at the jet edge, which is indicative of the high ordering of the poloidal magnetic field at the jet edges, and is lower in the interior, which is indicative of the helical twisting of the field. In general, the fractional polarization declines rapidly beyond an axial distance of $27 R$ in simulation $\mathrm{A}$ and declines beyond $30 \mathrm{R}$ for the denser jets in simulations $B$ and $C$. However, a central spine is evident in simulation B out to about $42 R$ in both the total intensity and fractional polarization images and in simulation $\mathrm{C}$ out to about $54 R$. Low fractional polarization at large axial distances occurs as the line of sight passes through many zones with different magnetic field orientation, although the magnetic field may be ordered on size scales larger than one zone. The diagonal structures seen in the intensity image in simulation $\mathrm{C}$ are relatively highly polarized suggesting strong ordered magnetic fields in these filaments. The increase of fractional polarization at the outer edges of the magnetized sheath is caused by fewer zones contributing to the line-of-sight integration and may not indicate a higher level of organized structure in the magnetic field. However, the filamentary features and ripples at the outer edges of the sheath, which are seen in both the fractional polarization and intensity images of all three simulations, suggest some overall organization in fluid motions at the outer edges of the magnetized sheath.

At an axial distance of $48 R$, the fractional polarization images show that magnetized jet material spans a distance of $\sim 10 R, \sim 8.5 R$, and $\sim 8 R$, and the intensity images indicate a distance of $\sim 8.5 R, \sim 7 R$, and $\sim 5 R$ in simulations $A$, $\mathrm{B}$, and $\mathrm{C}$, respectively. The difference in the sizes indicates a reduction in mixing and an increasing central concentration as the jet density is increased and the jet velocity is decreased. In fact, in simulation $C$, magnetized jet material appears to fill only a small portion of the cross section at $48 R$ because the amount of magnetized material and the associated magnetic field and magnetic pressure are so low far from the central concentration.

Organized structures found in the line of sight and cross section images are summarized below.

1. In simulation $\mathrm{A}$, images show evidence for pinching $\left(\lambda_{p} / R \sim 6\right)$, helical twisting $\left(\lambda_{h} / R \sim 20\right)$, and elliptical distortion $\left(\lambda_{e} / R \sim 12\right)$. 

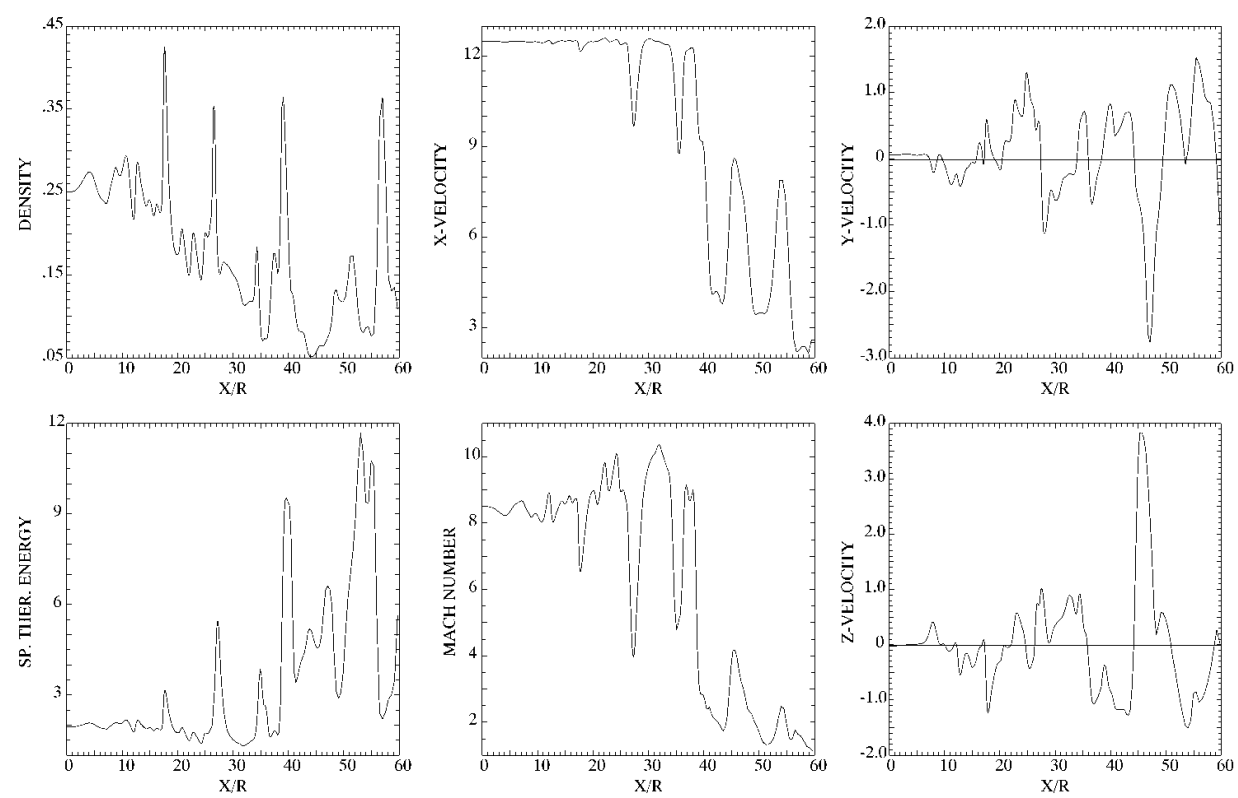

Fig. 6.-Axial profiles of the density, specific thermal energy, sonic Mach number, $M_{\mathrm{jt}} \equiv u / a_{\mathrm{jt}}$, and the three velocity components scaled relative to the sound speed, $a_{\mathrm{ex}}$, in the external medium in simulation A.

2. In simulation $B$, images show evidence for helical twisting $\left(\lambda_{h} / R \sim 12\right)$, internal transverse oscillation $\left(\lambda_{h} / R \sim\right.$ $5)$, elliptical distortion $\left(\lambda_{e} / R \sim 13.5\right)$, and internal dual twisted filaments that we identify with elliptical distortion $\left(\lambda_{e} / R \sim 4.5\right)$.

3. In simulation $\mathrm{C}$, images show evidence for helical twisting $\left(\lambda_{h} / R \sim 14\right)$, elliptical distortion $\left(\lambda_{e} / R \sim 14\right)$, internal dual twisted filaments $\left(\lambda_{e} / R \sim 4\right)$, triangular distortion $\left(\lambda_{t} / R \sim 9\right)$, and square distortion $\left(\lambda_{s} / R \sim 6\right)$.

4. All structures move with a comparable but not identical speed with $v_{w} \approx 0.30 u, v_{w} \approx 0.54 u$, and $v_{w} \approx 0.74 u$ in simulations $\mathrm{A}, \mathrm{B}$ and $\mathrm{C}$, respectively.

\subsection{Structure along the Jet Axis}

Axial profiles of density, temperature, sonic Mach number $\left(M_{\mathrm{jt}}\right)$, and the three velocity components are shown for simulations A, B, and C in Figures 6, 7, and 8, respectively. The axial velocity remains constant out to axial distances of $\sim 27 R, \sim 29 R$, and $\sim 32 R$ in simulations $\mathrm{A}, \mathrm{B}$, and $C$, respectively, after which there are one or more significant dips. Temperature and density increases associated with the dips suggest that these features are shocks. The dips appear periodically with wavelengths of $\sim 8 R, \sim 9 R$, and $\sim 14 R$, in simulations A, B, and C, respectively. Several bright knots or filaments in the intensity images can be associated with the dips in the axial velocity plots. For example, a bright knot at $\sim 27 R$ in simulation A (see Fig. 2), one at $\sim 29 R$ in simulation B (see Fig. 3), and the diagonal filaments at $\sim 33 R$ and $\sim 45 R$ in simulation $C$ (see Fig. 5) are at the locations of dips in the axial velocity. The dips are accompanied by a decrease in the average velocity, which falls off more slowly as the jet density is increased. Typically, the
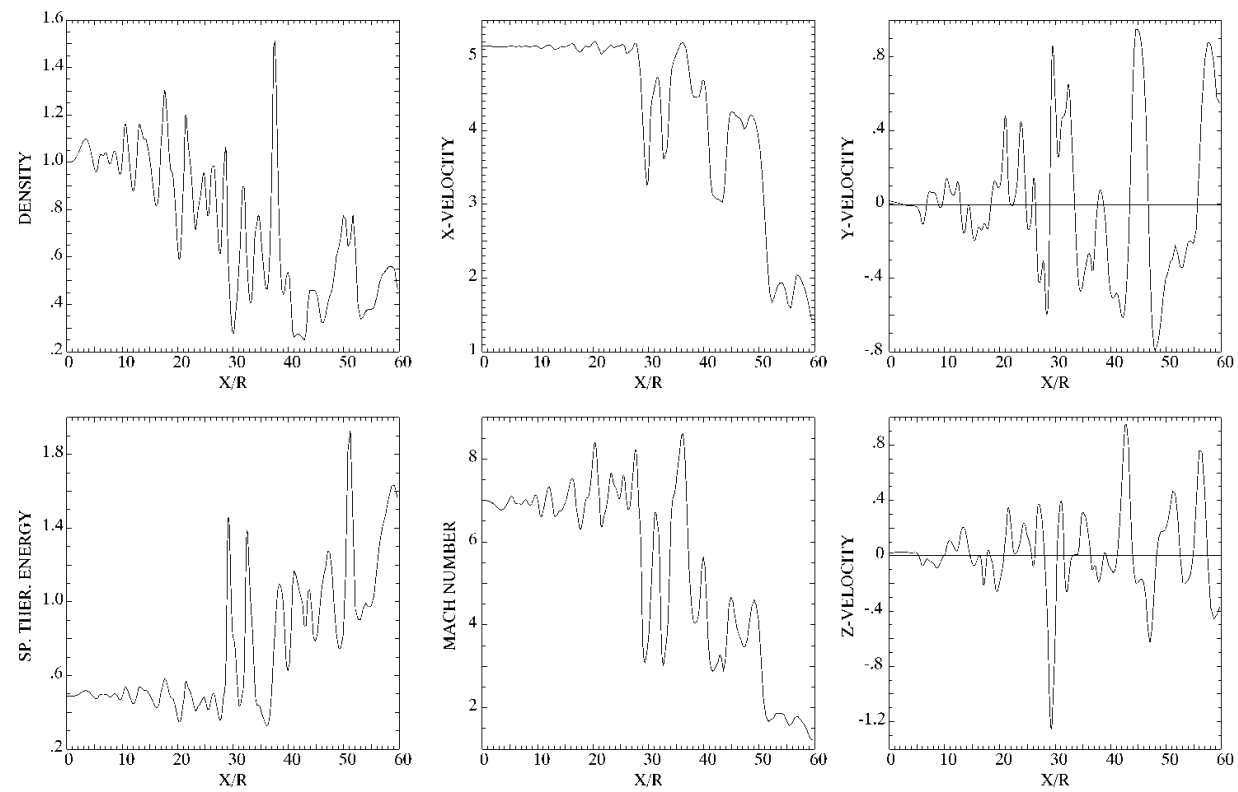

FIG. 7.-Same as Fig. 6 but for simulation B 

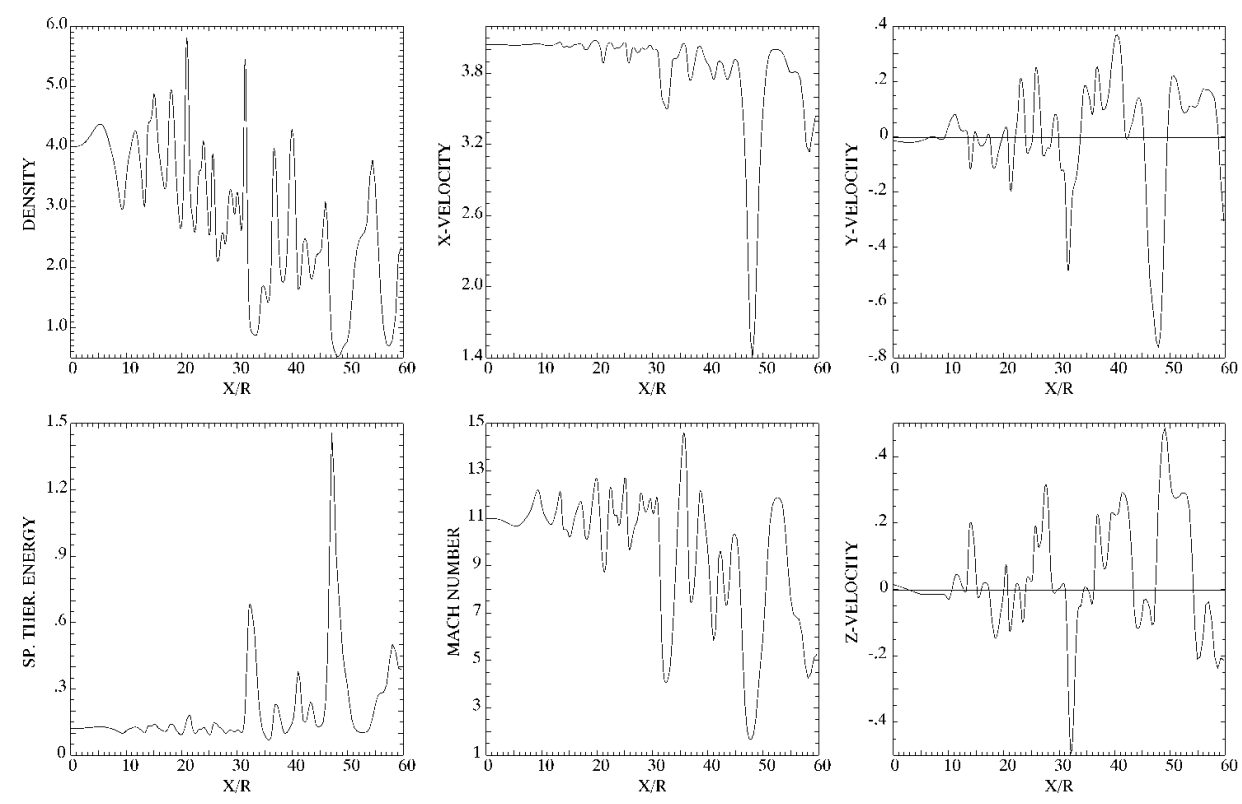

FIG. 8.-Same as Fig. 6 but for simulation $\mathrm{C}\left(\tau_{d}=36\right)$

decrease in average jet velocity is accompanied by an increase in average temperature, and a decrease in the sonic Mach number. At large axial distances, the light and equal density jets are reduced to transonic and mildly supersonic speeds, respectively, while the dense jet remains highly supersonic. The axial profiles also show smaller scale axial velocity oscillations, which are most evident in the Mach number plot. These oscillations have wavelengths $\sim 1 R$ and $\sim 2 R$ at axial distances in the ranges $\sim 13 R-17 R$ and $\sim 19 R-26 R$, respectively, in simulation $A$, and at axial distances in the ranges $\sim 5 R-10 R$ and $\sim 20 R-27 R$, respectively, in simulation B. Somewhat longer wavelengths, $\sim 1.5 R$ and $\sim 3.5 R$, are found in the ranges $\sim 23 R-30 R$ and $\sim 32 R-45 R$, respectively, in simulation C.

The axial plots also reveal that the transverse velocity contains oscillatory structure at many different wavelengths: $\sim 2 R, \sim 4 R$, and $\sim 20 R$ in simulation $\mathrm{A} ; \sim 1.5 R$, $\sim 3 R$ and $\sim 12 R$ in simulation $\mathrm{B} ; \sim 1.5 R, \sim 3 R$, and $\sim 14 R$ in simulation C. At the longest wavelength, the orthogonal transverse velocity components are out of phase and are associated with helical twisting of the jet. In simulation B, there is also evidence for transverse oscillations at a wavelength of $6 R-7 R$ between axial distances of $30 R$ and $50 R$. Typical transverse velocities are $\sim 0.75 a_{\mathrm{ex}}\left(\sim 0.4 a_{\mathrm{jt}}^{\mathrm{ms}}\right)$, $\sim 0.4 a_{\text {ex }}\left(\sim 0.4 a_{\mathrm{jt}}^{\mathrm{ms}}\right)$, and $\sim 0.2 a_{\mathrm{ex}}\left(\sim 0.4 a_{\mathrm{jt}}^{\mathrm{ms}}\right)$ in simulations $\mathrm{A}, \mathrm{B}$, and $\mathrm{C}$, respectively. Maximum transverse velocities are $\sim 4 a_{\text {ex }}\left(\sim 2 a_{\mathrm{jt}}^{\mathrm{ms}}\right), \sim a_{\mathrm{ex}}\left(\sim a_{\mathrm{jt}}^{\mathrm{ms}}\right)$, and $\sim 0.8 a_{\mathrm{ex}}\left(\sim 1.5 a_{\mathrm{jt}}^{\mathrm{ms}}\right)$ in simulations $\mathrm{A}, \mathrm{B}$, and $\mathrm{C}$, respectively. In simulation $\mathrm{A}$, the maximum transverse velocity is comparable to the axial velocity.

\subsection{Structure Transverse to the Jet Axis}

Transverse profiles of density, magnetic pressure, and axial velocity along the $y$-axis at axial distances from $12 R$ to $48 R$ in $12 R$ increments are shown in Figures 9, 10, and 11 for simulations $\mathrm{A}, \mathrm{B}$, and $\mathrm{C}$ (at $\tau_{d}=36$ ). Note that the vertical scales are not constant. In simulations $A$ and $B$, the typical magnetic pressure declines by over factors of 4 and 3 , respectively, while typical jet densities decline by no more than about factors of 4 and 3, respectively. As a result, the typical Alfvén speed decreases slightly, and Alfvénic Mach number, $M_{\mathrm{jt}}^{\mathrm{A}}$, increases slightly with axial distance. Thus, the light jet is transmagnetosonic and transonic, and the equal density jet is weakly supermagnetosonic and weakly supersonic at large axial distances. In simulation $\mathrm{C}$, the typical magnetic pressure and density vary only slightly and in a similar fashion, and the slight drop in axial velocity leads to the heavy jet remaining supermagnetosonic and supersonic at large axial distances.

By an axial distance of $12 R$, mixing of jet and external fluid has begun in all simulations. The dense jet is still highly collimated, although acceleration of material external to the jet to velocities $\sim 1.5 a_{\mathrm{ex}}$ is suggested by the velocity profile. That there has been mixing between the fluids is suggested by the small magnetic field in fluid outside the initial jet radius. The accelerations (and associated magnetic fields beyond $1 R$ ) are smaller in the $12 R$ profiles for the light and equal density jets. At somewhat larger axial distances, some of the asymmetries in the density and magnetic pressure profiles are related to the growth of helical twisting.

Farther down the jet at an axial distance of $24 R$, the magnetic pressure profiles show that magnetized jet material spans a distance of $\sim 6 R, \sim 5 R$, and $\sim 4 R$ in simulations A, B, and C, respectively. At an axial distance of $48 R$, the magnetic pressure profile spans a distance of $\sim 10 R, \sim 7 R$, and $\sim 6 R$ in simulations $\mathrm{A}, \mathrm{B}$, and $\mathrm{C}$, respectively. In simulation $A$, the spread of magnetized jet material in the transverse profile corresponds relatively closely to the spread in the fractional polarization image (see Fig. 2). In simulations B and C, the spread of magnetized jet material suggested by the transverse profile corresponds more closely to the spread in the intensity image than the fractional polarization image (see Figs. 3 and 5) and is evidence for a more centrally concentrated jet. In simulation $\mathrm{C}$, the density, magnetic pressure, and velocity profiles indicate a central spine with diameter comparable to the initial diameter of the jet.

At an axial distance of $48 R$, the transverse axial velocity profile shows that the outer portion of the magnetized sheath in simulation A is nearly at rest and that on average 

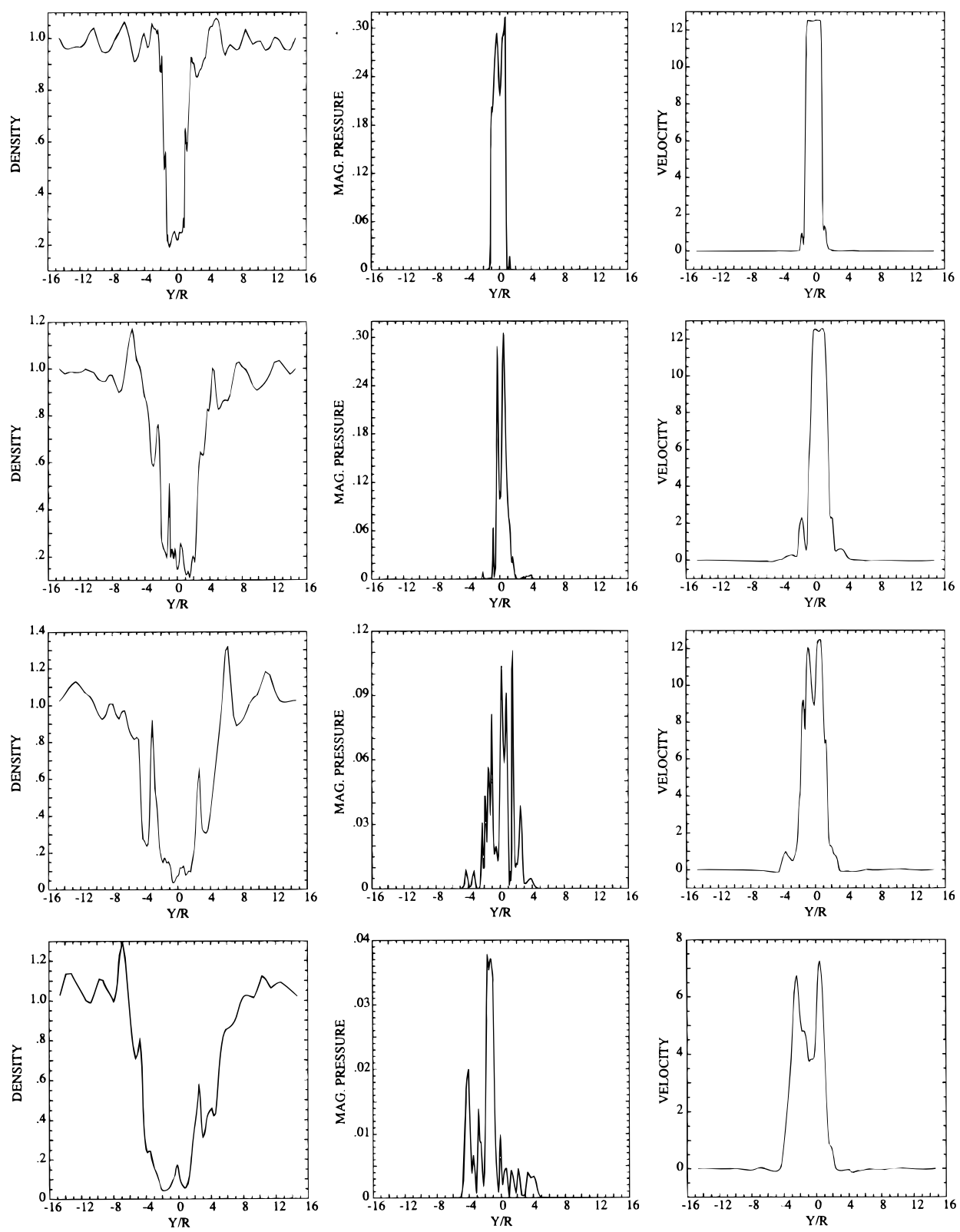

FIG. 9.-Transverse profiles along the $y$-axis of density (left-hand column), magnetic pressure (center column), and axial velocity (right-hand column) at axial distances of $12 R, 24 R, 36 R$, and $48 R$ from top to bottom rows in simulation $\mathrm{A}$. The density is scaled relative to the ambient density in the external medium, the magnetic pressure is in units such that $B^{2} / 2$ is pressure units, and the velocity is scaled relative to the sound speed, $a_{\mathrm{ex}}$, in the external medium.

the outward velocity has dropped to less than half the velocity at the inlet. The transverse axial velocity profile in simulation B shows that the velocity is $\geq 50 \%$ of the value at the inlet within a jet diameter and $\sim 80 \%$ of the value at the inlet within a spine of width on the order of a jet radius. The velocity profile also suggests that most of the magnetized sheath has a velocity $\approx 0.5 a_{\mathrm{ex}}$. In simulation $\mathrm{C}$, the transverse velocity and magnetic profiles suggest a significant sheath. The magnetized sheath approximately corresponds to the region in which flow speeds exceed the external sound speed. At larger transverse distances, outward flow is $\leq 0.5 a_{\mathrm{ex}}$, and the lack of magnetic field suggests that jet fluid has not mixed with external fluid at these larger transverse distances.

In simulations $\mathrm{A}$ and $\mathrm{B}$, considerable heating and expansion have accompanied mixing of jet and external material. In simulation $\mathrm{A}$ at and axial distance of $48 R$, the density over the region (diameter of about $4 R$ ) in which the velocity exceeds 3 times the external sound speed (roughly half the maximum speed at this distance) is typically less than half the jet density at the inlet. Outside the magnetized sheath, the medium has a density significantly below the ambient external density. In simulation $\mathrm{B}$ at $48 R$, the density has decreased by about a factor of 3 within the jet spine, and the density is typically decreased by about a factor of 2 within the magnetized sheath from the jet density at the inlet. Up to a distance of $2 R$ outside the magnetized sheath, the density remains below $80 \%$ of the initial jet and ambient density. In simulation $\mathrm{C}$, there appears to have been very little heating or expansion from mixing of jet and ambient fluid. Within the magnetized sheath, the density is comparable to the initial density in the undisturbed external medium.

All of these simulations, which initially have a strong poloidal magnetic field, show some evidence for filamentation, and this is most easily seen in the dense jet simulation. 

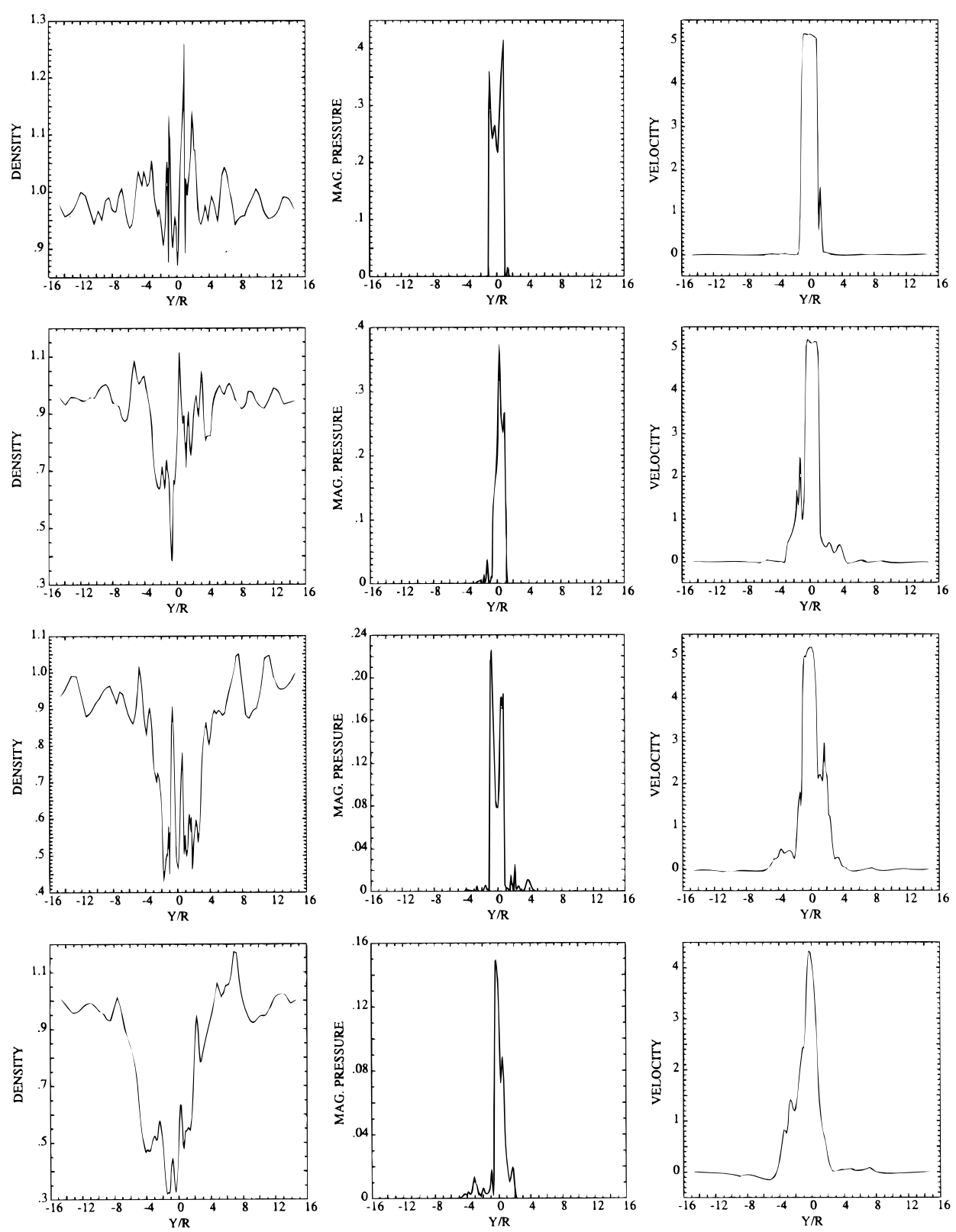

Fig. 10. - Same as Fig. 9 but for simulation B

In Figure 11 at an axial distance of $36 R$, the magnetic pressure and velocity profiles show some filamentation into multiple streams outside the central spine. At $48 R$, the profiles indicate further filamentation into multiple streams, and the magnetic field, largely concentrated in one of the streams, is very narrow along the $y$-axis. This narrowing is the reason for the sharp narrow maximum in the intensity image (see Fig. 5) at this distance. This stream still maintains the initial jet speed.

\subsection{Simulation Summary and Discussion}

In the linear regime close to the inlet, all three jets maintain relatively well organized surface and internal structure on many length scales both in the images and in the velocity plots. These structures include fluting of the jet surface and helically twisted bright filaments at the jet surface (possibly associated with the surface fluting) and in the jet interior and pinching in the jet interior. At least approximately, smaller scale structures appear nearest to the inlet with larger scale structures appearing farther from the inlet. In these simulations, all conducted with comparable magnetosonic Mach number, the linear development is similar. The smaller scale lesser amplitude structures disappear as larger scale structures grow to larger amplitudes farther from the inlet. All features propagate with comparable but not exactly the same "wave" speed.

The jets in these simulations propagate about halfway across the computational grid before filamentation and entrainment, in these simulations associated with jet flattening or helical twisting, become important. As the jet density increases, the jet propagates to larger distances before these nonlinear effects occur. Based on purely theoretical predictions from a linear analysis (see the following sections) the light jet (simulation A) with $M_{\mathrm{jt}}^{\mathrm{ms}} \sim 6$ should extend about $21 \%$ farther, and the heavy jet (simulation $C$ ) with $M_{\mathrm{jt}}^{\mathrm{ms}} \sim 7.7$ should extend about $57 \%$ farther than the equal density jet (simulation B) with $M_{\mathrm{jt}}^{\mathrm{ms}} \sim 4.9$ before nonlinear effects associated with large-scale structures become 

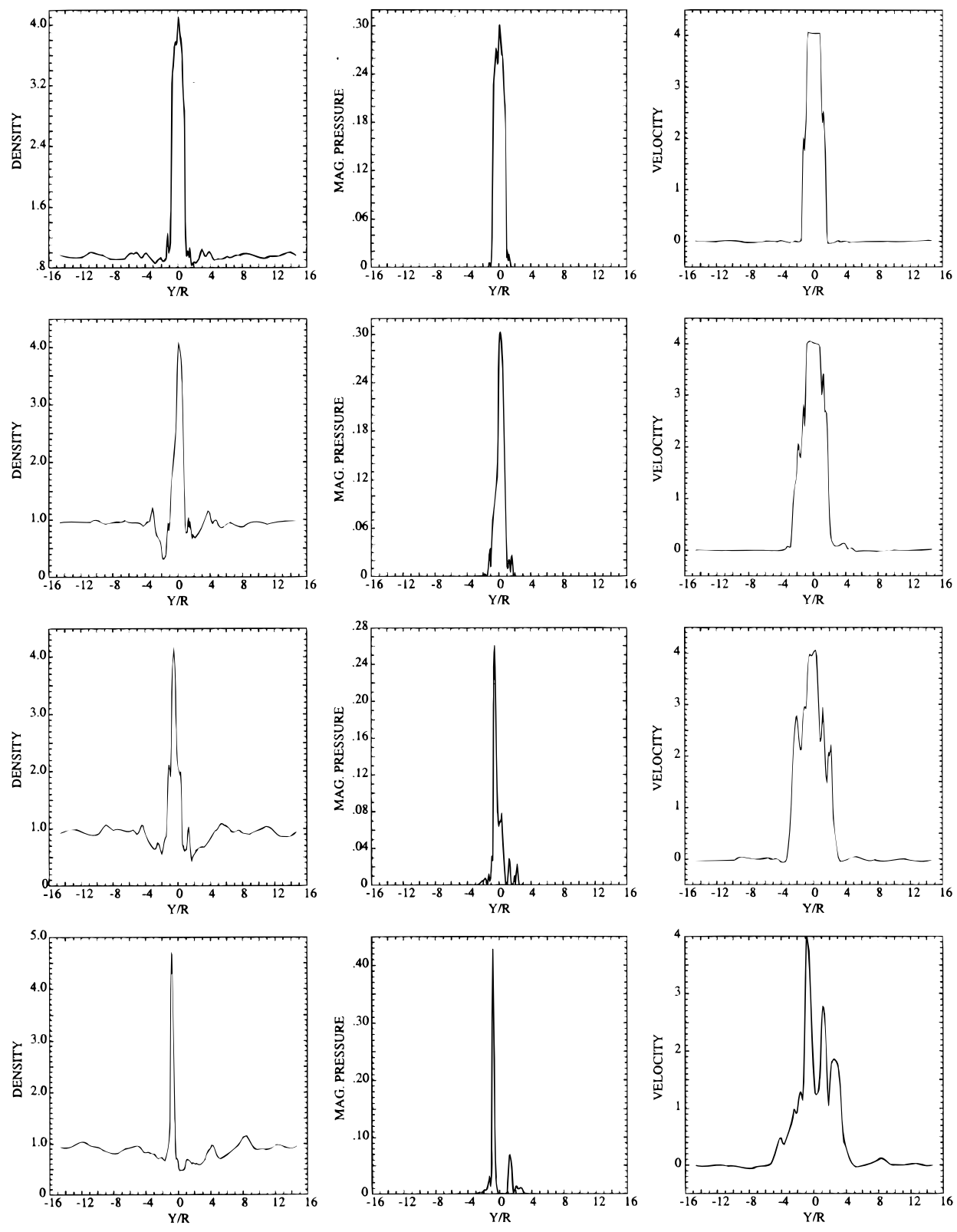

FIG. 11. - Same as Fig. 9 but for simulation $\mathrm{C}\left(\tau_{d}=36\right)$

important. In fact, the light jet does not extend quite as far as the equal density jet, and the heavy jet extends significantly farther than might be naively expected before the jet shows signs of slowing significantly. That is, the typical axial velocity remains nearly constant out to $\sim 27 R, \sim 29 R$, and $\sim 48 R$ in simulations A, B, and C, respectively.

When the light jet loses organized internal structure about halfway across the computational grid, it assumes a plumelike appearance with some residual oscillation associated with helical jet twisting excited by the precessional motion at the inlet. Transverse motions in the "plume" are typically submagnetosonic and no more than one-fifth of the axial motion. Occasionally, however, transverse motions are transmagnetosonic and comparable to the outward motion. On the other hand, the heavy jet develops a reasonably pronounced spine-sheath morphology in which a jet spine maintains the initial jet speed and is surrounded by a more slowly moving sheath. The jet spine maintains large-scale structure, e.g., jet flattening and helical twisting, across the entire computational grid. The equal density jet has a structure that is midway between the light and heavy jets. We find at the outer boundary of the computational grid that the maximum flow speed of the light, equal density, and heavy jets is approximately $50 \%$, $80 \%$, and $100 \%$ of the speed at the inlet, respectively.

A control simulation, parameters like simulation $\mathrm{C}$, has been performed with a precession frequency of $\omega R / a_{\mathrm{ex}}=1$ and a transverse velocity component of $10^{-6} u$. This precession provides just enough perturbation to smooth the jet boundary on the Cartesian grid but negligible angular momentum. In this case, the jet remains essentially axisymmetric across the computational grid with complex internal structure. Thus, the organized helical structures that we see in the present simulations do not occur in the absence of a significant precessional motion at the inlet.

Other numerical simulations have revealed that there is no significant dynamical difference between a purely poloidal magnetic field configuration and the present magnetic 
field configuration. Additionally, only small differences are seen in simulations with weaker poloidal magnetic fields. In general, numerical simulations indicate that a poloidal magnetic field has a relatively small although not negligible influence on the dynamics and stability of the collimated flow at a constant supermagnetosonic Mach number and constant density ratio. On the other hand, the inclusion of a strong toroidal magnetic field has been found to have a significant influence on jet dynamics. In general, the inclusion of a strong toroidal magnetic field appears to inhibit the development of the internal structures and the flattening and fluting surface distortions seen in the present poloidal magnetic field simulations. Accompanying the inhibition of these features is an inhibition in filamentation and a reduction in entrainment (Rosen et al. 1997a). Thus, numerical simulations indicate that the inclusion of strong toroidal field has a stabilizing influence (Rosen et al. 1997b).

\section{JET STABILITY THEORY AND JET STRUCTURE}

The stability of a poloidally magnetized cylindrical jet with top hat profile residing in a uniform unmagnetized medium has been investigated in several articles (Ray 1981; Ferrari, Trussoni, \& Zaninetti 1981; Fiedler \& Jones 1984; Bodo et al. 1989). For supermagnetosonic jets, increased poloidal magnetic field strength leads to only a very slight stabilization relative to a purely fluid jet. Strong toroidal magnetic fields, e.g., toroidal magnetic fields of strength comparable to the present equipartition poloidal magnetic fields, can influence the stability properties significantly and can lead to increased jet stability (Appl \& Camenzind 1992). However, inclusion of a small nonuniform toroidal magnetic field component such as is present in these numerical simulations does not significantly modify results obtained from the following linear stability analysis incorporating only poloidal magnetic fields.

In cylindrical geometry, a random perturbation to the equilibrium jet can be considered to consist of Fourier components of the form $f_{1}(r, \phi, x)=f_{1}(r) \exp [i(k x \pm n \phi$ $-\omega t)]$, where the flow is along the $x$-axis. These components propagate as waves with a dispersion relation given by equation (A6) in the Appendix. The normal modes of a cylindrical jet involve pinch $(n=0)$, helical $(n=1)$, elliptical $(n=2)$, triangular $(n=3)$, rectangular $(n=4)$, etc. distortions of the jet. In cylindrical geometry, $n$ is an integer and represents the azimuthal wavenumber. For $n>0$, waves propagate at an angle to the flow direction, and $+n$ and $-n$ specify wave propagation in the clockwise and counterclockwise sense, respectively, when viewed outward along the flow direction.

\subsection{The Low-Frequency Limit}

Each wave mode $n$ contains a surface wave and multiple body wave solutions to the dispersion relation. In the lowfrequency limit, the real part of the pinch mode $(n=0)$ surface wave solution becomes (Hardee 1995)

$$
\begin{aligned}
\frac{\omega}{k} \approx & u \pm \llbracket \frac{1}{2}\left[V_{\mathrm{A}, \mathrm{jt}}^{2}+\frac{V_{\mathrm{A}, \mathrm{jt}}^{2} a_{\mathrm{jt}}^{2}}{\left(a_{\mathrm{jt}}^{\mathrm{ms}}\right)^{2}}\right] \\
& \left. \pm \frac{1}{2}\left\{\left[V_{\mathrm{A}, \mathrm{jt}}^{2}+\frac{V_{\mathrm{A}, \mathrm{jt}}^{2} a_{\mathrm{jt}}^{2}}{\left(a_{\mathrm{jt}}^{\mathrm{ms}}\right)^{2}}\right]^{2}-4 \frac{V_{\mathrm{A}, \mathrm{jt}}^{4} a_{\mathrm{jt}}^{2}}{\left(a_{\mathrm{jt}}^{\mathrm{ms}}\right)^{2}}\right\}^{1 / 2}\right] 1 / 2,
\end{aligned}
$$

where $a_{\mathrm{jt}}^{\mathrm{ms}} \equiv\left(a_{\mathrm{jt}}^{2}+V_{\mathrm{A}, \mathrm{jt}}^{2}\right)^{1 / 2}$ is the magnetosonic speed, $a_{\mathrm{jt}}=$ $\left(\Gamma p_{\mathrm{jt}} / \rho_{\mathrm{jt}}\right)^{1 / 2}$ is the jet sound speed, and $V_{\mathrm{A}, \mathrm{jt}}=\left(B_{\mathrm{jt}}^{2} / 4 \pi \rho_{\mathrm{jt}}\right)^{1 / 2}$ is the jet Alfvén speed. The imaginary part of the solution is vanishingly small in the low-frequency limit. These solutions are related to fast $(+)$ and slow $(-)$ magnetosonic waves propagating with $(u+)$ and against $(u-)$ the jet flow speed $u$ but are strongly modified by the jet-external medium interface. The unstable growing solution is associated with the backward-moving (in the jet fluid reference frame) slow magnetosonic wave and in the supermagnetosonic limit moves at nearly the flow speed in the observer frame.

In this limit, all higher order modes $(n>0)$ have surface wave solutions given by

$$
\frac{k u}{\omega} \approx \frac{1}{1-V_{\mathrm{A}, \mathrm{jt}}^{2} / u^{2}}\left\{1 \pm i\left[\left(1-\frac{V_{\mathrm{A}, \mathrm{jt}}^{2}}{u^{2}}\right) \frac{1}{\eta}-\frac{V_{\mathrm{A}, \mathrm{jt}}^{2}}{u^{2}}\right]^{1 / 2}\right\},
$$

where $\eta \equiv \rho_{\mathrm{jt}} / \rho_{\mathrm{ex}}$. Spatial growth corresponds to the minus sign in equation (2) and a negative value for the imaginary part of the complex wavenumber. In the dense jet limit, i.e., $\eta \rightarrow \infty$, equation (2) becomes $\omega / k \approx u \pm V_{\mathrm{A}, \mathrm{j}}$, and the surface waves are related to Alfvén waves propagating with and against the jet flow speed $u$ but are strongly modified by the jet-external medium interface. The unstable growing solution is associated with the backward-moving (in the jet fluid reference frame) Alfvén wave. In the supermagnetosonic limit, the real part of $\omega / k \approx[\eta /(1+\eta)] u$ in the observer frame and the wave speed is a strong function of the density ratio.

In this limit, the body wave solutions are purely real, nonpropagating, and are given by

$$
k R=\frac{(n+2 m-1 / 2) \pi / 2}{\llbracket\left\{\left(M_{\mathrm{jt}}^{\mathrm{ms}}\right)^{2} /\left[1-\left(M_{\mathrm{jt}}^{\mathrm{ms}} / M_{\mathrm{jt}} M_{\mathrm{jt}}^{\mathrm{A}}\right)^{2}\right]\right\}-1 \rrbracket^{1 / 2}},
$$

where $n$ is the mode number, and $m \geq 1$ is the integer body mode number. Additionally, $M_{\mathrm{jt}} \equiv u / a_{\mathrm{jt}}$ is the sonic Mach number, $\boldsymbol{M}_{\mathrm{jt}}^{\mathrm{A}} \equiv u / V_{\mathrm{A}, \mathrm{jt}}$ is the Alfvén Mach number, and $M_{\mathrm{jt}}^{\mathrm{ms}} \equiv u / a_{\mathrm{jt}}^{\mathrm{ms}}$ is the magnetosonic Mach number.

\subsection{The Maximum Growth Rate}

With the exception of the pinch mode surface wave, which has a relatively broad plateau in the growth rate, all other solutions have a relatively sharp maximum in the growth rate when a jet is supermagnetosonic. In the supermagnetosonic limit, a maximum in the growth rate is achieved for

$$
\begin{gathered}
\omega R / a_{\mathrm{ex}} \approx \omega_{n m}^{*} R / a_{\mathrm{ex}} \equiv(n+2 m+1 / 2) \pi / 2, \\
\lambda \approx \lambda_{n m}^{*} \equiv \frac{2 \pi}{\omega_{n m}^{*} R / a_{\mathrm{ex}}} \frac{M_{\mathrm{jt}}^{\mathrm{ms}}}{1+M_{\mathrm{jt}}^{\mathrm{ms}} / M_{\mathrm{ex}}} R, \\
v_{w} \approx v_{w}^{*} \equiv \frac{M_{\mathrm{jt}}^{\mathrm{ms}} / M_{\mathrm{ex}}}{1+M_{\mathrm{jt}}^{\mathrm{ms}} / M_{\mathrm{ex}}} u,
\end{gathered}
$$

where $n$ gives the wave mode, $m=0$ gives the surface wave, and $m \geq 1$ gives the body waves. The wave speed at the maximum growth rate is significantly different from the low frequency wave speed.

With the exception of the $n=0, m=0$, pinch mode surface wave, the maximum spatial growth rate is approximately given by

$$
k_{I} \approx k_{I}^{*} \equiv-\left(2 M_{\mathrm{jt}}^{\mathrm{ms}} R\right)^{-1} \ln \left(4 \frac{\omega_{n m}^{*} R}{a_{\mathrm{ex}}}\right),
$$



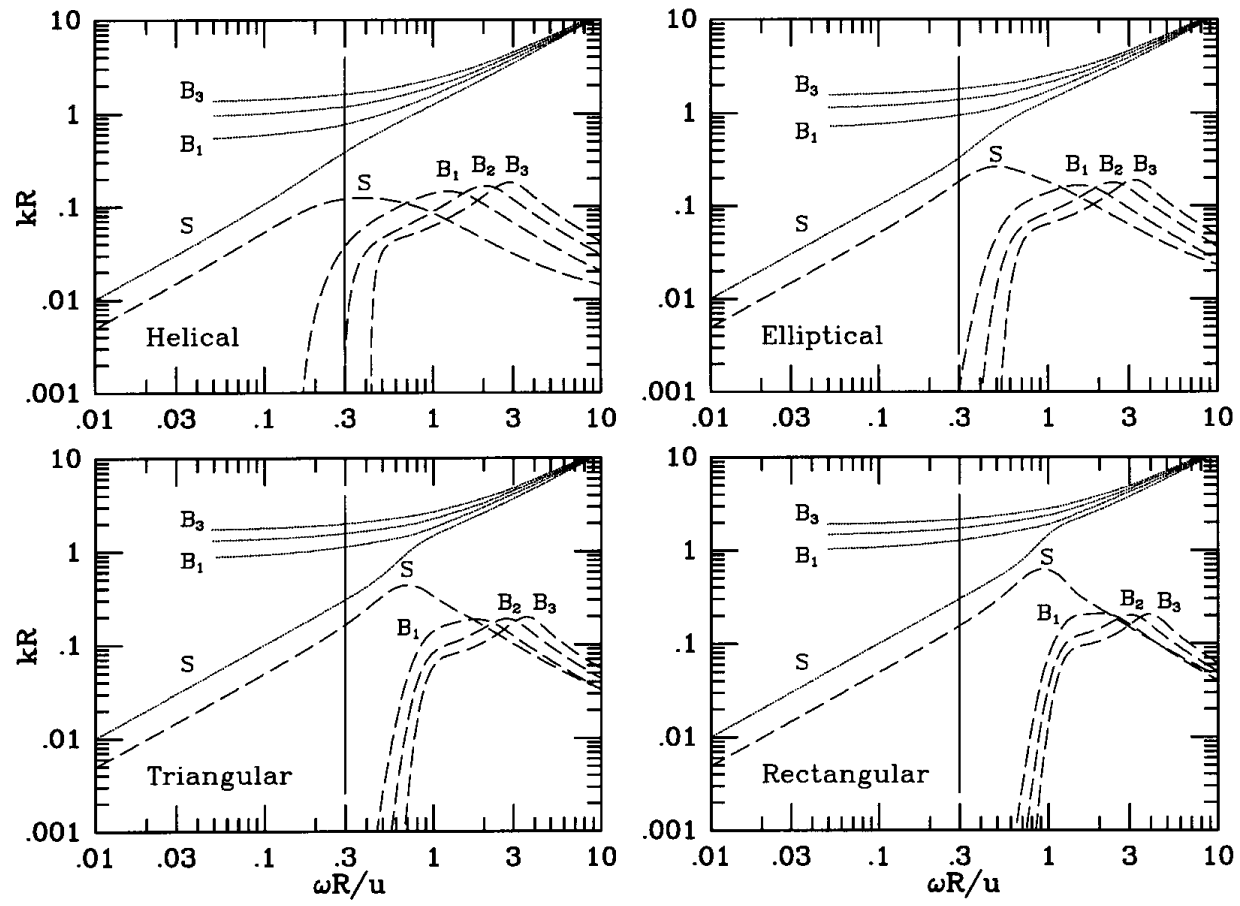

FIG. 12.-Numerical solution of the dispersion relation for parameters appropriate to simulation C. The surface $(S)$ wave and first three-body $\left(B_{1}, B_{2}\right.$, $\left.B_{3}\right)$ wave solutions are shown for the helical, elliptical, triangular and rectangular modes. The dotted lines give the real part of the wavenumber, $k_{R}$, and the dashed lines give the absolute value of the imaginary part of the wavenumber, $\left|k_{I}\right|$.

where $k_{I}$ is the imaginary part of the complex wavenumber. When a jet is transmagnetosonic but super-Alfvénic, the growth rate of the helical and higher order surface waves has no maximum but increases proportional to the frequency, and the growth rate of the body waves decreases. When a jet is sub-Alfvénic, the helical and higher order surface waves are stable (Bodo et al. 1989; Hardee et al. 1992a), but the pinch surface wave can remain unstable. Body waves exist and are unstable provided the denominator of equation (3) is real, and this occurs if the jet speed exceeds the fast magnetosonic speed or if the jet speed is slightly below the slow magnetosonic speed (Bodo et al. 1989; Hardee et al. 1992a).

\subsection{The High-Frequency Limit}

In the high-frequency limit, the real part of the solutions to the dispersion relation for all surface and body waves is given by

$$
\left.\frac{\omega}{k} \approx u \pm \llbracket \frac{1}{2}\left(a_{\mathrm{jt}}^{2}+V_{\mathrm{A}, \mathrm{jt}}^{2}\right)\left\{1 \pm\left[1-\frac{4 a_{\mathrm{jt}}^{2} V_{\mathrm{A}, \mathrm{jt}}^{2}}{\left(a_{\mathrm{jt}}^{2}+V_{\mathrm{A}, \mathrm{jt}}^{2}\right)^{2}}\right]^{1 / 2}\right\}\right] 1 / 2 .
$$

Equation (6) describes fast, $\omega / k \approx u \pm \operatorname{Max}\left(V_{\mathrm{A}, \mathrm{j} t}, a_{\mathrm{jt}}\right)$, and slow, $\omega / k \approx u \pm \operatorname{Min}\left(V_{\mathrm{A}, \mathrm{jt}}, a_{\mathrm{jt}}\right)$, magnetosonic waves. The unstable growing solution is associated with the backwardmoving (in the jet fluid reference frame) fast magnetosonic wave, but the growth rate is vanishingly small in the highfrequency limit. The wave speed in the observer frame is again considerably different from the wave speed at low frequency and at the maximum growth rate.

\subsection{Numerical Solutions}

We have solved the dispersion relation numerically using root-finding techniques over a wide range of perturbation frequencies for parameters appropriate to the three numerical simulations. Figure 12 shows the numerical solution for the surface and first three-body waves associated with the helical $(n=1)$, elliptical $(n=2)$, triangular $(n=3)$, and rectangular $(n=4)$ modes for parameters appropriate to the heavy jet in simulation $C$, and Table 2 contains the wavelengths at the maximum growth rate. The body waves have a larger maximum growth rate than the surface wave only for the pinch (not shown) and helical modes. We note that the maximum growth rate of the $n>1$ surface wave modes exceeds the maximum growth rate given by equation (5) but that the computed maximum growth rate of all body wave modes remains below the maximum predicted by equation (5). The vertical line in the panels is at the precessional perturbation frequency used in simulation C. Numerical solutions for parameters appropriate to the other two simulations are qualitatively similar. Quantitative differences between numerical solution of the dispersion relation for simulations A and B occur largely in the maximum growth rate as a result of the slightly different magnetosonic Mach numbers and in the wavelength as a function of frequency as a result of different wave propagation speeds for the different density jets.

The displacement and velocity perturbations associated with the normal modes of a cylindrical jet can be written in

TABLE 2

Wavelengths at Maximum Growth in Simulation C

\begin{tabular}{crccc}
\hline \hline Mode & $\lambda_{\mathrm{S}}^{*} / R$ & $\lambda_{\mathrm{B} 1}^{*} / R$ & $\lambda_{\mathrm{B} 2}^{*} / R$ & $\lambda_{\mathrm{B} 3}^{*} / R$ \\
\hline Pinch ............ & \multicolumn{1}{c}{$\ldots$} & 4.9 & 2.5 & 1.7 \\
Helical .......... & 12.5 & 3.4 & 2.0 & 1.4 \\
Elliptical ........ & 9.2 & 2.7 & 1.7 & 1.3 \\
Triangular ....... & 6.5 & 2.3 & 1.5 & 1.2 \\
Rectangular ...... & 4.8 & 2.0 & 1.3 & 1.0 \\
\hline
\end{tabular}


the form (see Appendix)

$$
\begin{gathered}
\xi\left(r, \phi_{s}, x_{s}\right)=\boldsymbol{A}(r) e^{i \Delta(r)} \xi_{r, n}(R) \exp \left[i\left(k x_{s} \pm n \phi_{s}-\omega t\right)\right], \\
\boldsymbol{u}_{1}=-i(\omega-k u) \boldsymbol{A}(r) e^{i \Delta(r)} \xi_{r, n}(R) \exp \left[i\left(k x_{s} \pm n \phi_{s}-\omega t\right)\right],
\end{gathered}
$$

where $\boldsymbol{u}_{1}(r, \phi, x)=d \xi / d t, \xi_{r, n}(R)$ is the surface amplitude of the radial displacement, $x_{s}$ is the unperturbed axial location at the jet surface, $\phi_{s}$ is the azimuthal phase angle at the jet surface, and $\boldsymbol{A}(r) \leq 1$ and $\Delta(r)$ give the amplitude and phase dependence relative to the jet surface, respectively. If the dependence of the radial fluid displacement inside the jet on rotation in azimuth is ignored, then the radial fluid displacement of a surface wave mode $n$ is approximately given by $\xi_{r}(r) \approx\left[\xi_{r, n}(R)\right](r / R)^{n-1}$, and therefore displacements produced by higher order surface modes are predicted to show a much more rapid decrease inward (Hardee 1983). In fact the surface waves show a somewhat faster falloff in amplitude relative to the surface amplitude than that predicted by the analytical approximation as a result of significant rotation in azimuth of, say, the maximum internal displacement relative to the maximum surface displacement (Hardee et al. 1995). At a constant azimuth, the body waves show a reversal in fluid displacement at null surfaces inte- rior to the jet surface. Only the helical surface and body waves induce displacement of the jet center. Displacements produced by body waves are also, in general, largest at the jet surface and decrease inward (see Hardee et al. 1995).

Fluid displacement surfaces associated with the helical, elliptical, triangular, and rectangular mode surface and first three body waves computed from equation (7a) for parameters appropriate to the heavy jet simulation $\mathrm{C}$ at the appropriate maximally growing frequencies (see Fig. 12) are shown in Figure 13. Note how the distortion of inner surfaces becomes more out of phase with the jet surface as the jet center is approached for all surface and body modes but that little internal distortion accompanies the triangular and rectangular surface wave modes. Behavior appropriate to the simulations is primarily of the form $f_{1}(r, \phi, x)=$ $A(r) e^{i \Delta(r)} f_{1}(R) \exp \left[i\left(k x_{s}-n \phi_{s}-\omega t\right)\right]$, and over time $\left(x_{s}=\right.$ const, $n \phi_{s}+\omega t=$ const) patterns rotate in the counterclockwise direction (flow is into the page as in the magnetic pressure cross sections associated with the simulations). Thus, internal displacement surfaces exhibit a phase lead relative to the surface displacement, e.g., the innermost displacement surface shown associated with the helical surface and first body waves leads the surface displacement by about $30^{\circ}$ and $225^{\circ}$, respectively. The spiral patterns associated with the displacement surfaces are "trailing" as the patterns rotate in the counterclockwise direction at fixed
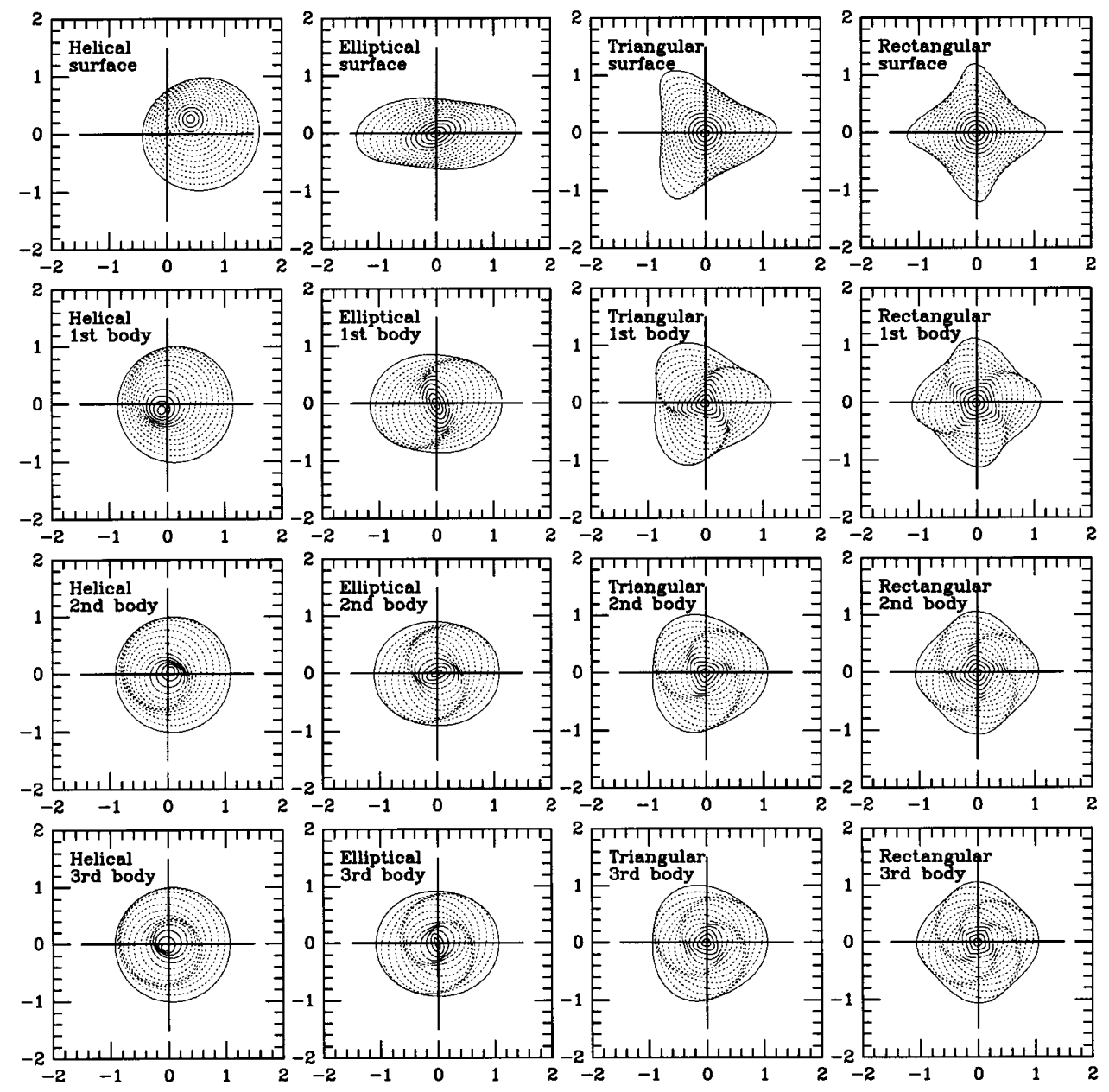

FIG. 13.-Fluid displacement surfaces accompanying the surface and first three body waves associated with the helical, elliptical, triangular, and rectangular modes. Displacement surfaces are computed for parameters appropriate to the heavy jet simulation $\mathrm{C}$ using the solutions to the dispersion relation shown in Fig. 12 at the maximum growth rates. 
position. Note, however, that as one moves out along the jet axis $\left(t=\right.$ const, $k_{R} x_{s}-n \phi_{s}=$ const), all patterns twist in a clockwise sense with a spatial rate $\phi_{s}^{n}(x)=\left(k_{R} / n\right) x_{s}$, where $k_{R}$ is the real part of the wavenumber.

In Figure 13, approximately the largest possible displacements of initially circular surfaces are shown to emphasize the distortions, and a maximum in the surface displacement is chosen to lie along the positive horizontal axis ( $z$-axis in the simulations). At least approximately, the maximum displacement amplitude must be less than that leading to the overlap of displacement surfaces computed from the linearized equations because overlap implies the development of shocks. Using this criterion, we find that the maximum amplitude associated with the maximum growth rate for the surface wave solutions to the helical, elliptical, triangular and rectangular modes is given by

$$
\left[\xi_{r, n}^{*}(R)\right]^{\max } \approx \frac{0.9}{n+1 / 2} R,
$$

and for the first three-body wave solutions to the pinch, helical, elliptical, triangular, and rectangular modes is given by

$$
\left[\xi_{r, n m}^{*}(R)\right]^{\max } \approx \frac{0.7}{n+2 m+1 / 2} R
$$

The maximum amplitudes are basically $\left[\xi_{r, n m}^{*}\right]^{\max } \propto$ $\left(\omega_{n m}^{*}\right)^{-1} \propto(n+2 m+1 / 2)^{-1}$, and maximum displacements become smaller for higher order surface waves (larger $n$ ) and higher order body waves (larger $m$ ). For longer or shorter wavelengths, i.e., waves at frequencies below or above maximum growth, larger or smaller maximum surface amplitudes are allowed with the maximum allowed amplitude

$$
\xi_{r, n m}^{\max }(R) \approx\left(\lambda_{n m} / \lambda_{n m}^{*}\right)\left(v_{w} / v_{w}^{*}\right)\left[\xi_{r, n m}^{*}(R)\right]^{\max },
$$

where $\lambda_{n m}^{*}$ and $v_{w}^{*}$ are the wavelength and wave speed at the maximum growth rate. These surface amplitudes require velocity perturbations transverse to the flow less than the internal jet magnetosonic speed and lesser amplitudes require velocity perturbations $u_{r 1}(R) / u_{r 1}^{\max }(R) \approx$ $\xi_{r, n m}(R) / \xi_{r, n m}^{\max }(R)$. At the maximum amplitudes, the perturbations are no longer linear.

\section{COMPARISON WITH SIMULATIONS}

The predicted stability properties of jets in the supermagnetosonic regime are (1) a very small growth rate for the pinch mode surface wave, (2) a slightly higher maximum growth rate for all the body wave modes compared to the maximum growth rate of the helical mode surface wave, and (3) a higher maximum growth rate for higher order surface waves compared to the maximum growth rate of all the body wave modes. The computed growth rates along with the maximum allowed displacements would lead us to predict that significant dynamical structures will be produced by a few of the lower order surface and body wave modes. Of these few modes, the higher order modes should achieve maximum displacements closer to the jet inlet as a result of the faster spatial growth rate, and the lower order modes should achieve larger maximum displacements farther from the jet inlet. Note that in the simulations, the inlet perturbation favors development of the helical surface mode and thus higher order body and surface modes may not develop as close to the inlet as might be expected if all wave modes were excited with the same initial amplitude. Nevertheless, in the simulations we see that the higher order modes develop first to some maximum amplitude and then decline in amplitude as the lower order modes grow to larger amplitudes. In simulations A and B, the larger amplitudes associated with the helical and elliptical modes lead to disruption of organized structure. We note that in each simulation, the propagation speed of surface and body wave modes is similar and is within $10 \%$ of the value computed from equation (4c). Interestingly, the wave speeds observed in the simulations (computed from the linear theory) are $0.30 u(0.32 u)$ from simulation A, $0.54 u(0.49 u)$ from simulation $\mathrm{B}$, and $0.74 u(0.66 u)$ from simulation $\mathrm{C}$. This reveals a definite trend toward observed speeds being higher than computed speeds as the jet density increases.

All simulations show similar structures in the linear limit. Because the structures are better defined in simulation C and grow to nonlinear amplitudes without disruption, we turn to comparison with simulation $\mathrm{C}$ to obtain nonlinear estimates of the maximum amplitudes associated with surface and body wave modes. In order to identify the surface modes responsible for structures observed in the numerical simulations, we have attempted to fit the welldefined jet surface distortions observed in cross sections in simulation $\mathrm{C}$ using displacement surfaces computed from equation (7a), and the results are shown in Figure 14. The displacement surfaces shown in Figure 14 were produced as a linear sum of displacements produced by helical, elliptical, triangular, and rectangular surface waves at the positions of the cross sections shown in Figure 4 at time $\tau_{d}=34$. No fit was attempted at an axial distance of $48 R$ as the jet had not yet reached a quasi-equilibrium configuration at this distance by this dynamical time. The cross sections can be fitted by (1) a helical distortion whose phase angle rotates through $360^{\circ}$ over a wavelength $\lambda_{1} \approx 14 R$ and which builds to a maximum surface amplitude of $\xi_{r 1}^{\text {obs }} / R \approx 0.8$ at an axial distance of $36 R$ and maintains this amplitude at larger distance; (2) an elliptical distortion that rotates through a phase angle of $180^{\circ}$ over a wavelength $\lambda_{2} \approx 14 R$ and which builds to a maximum surface amplitude of $\xi_{r 2}^{\text {obs }} / R \approx 0.6$ at an axial distance of $42 R$ and maintains this amplitude at larger distance; (3) a triangular distortion whose phase angle rotates through $120^{\circ}$ over a wavelength $\lambda_{3} \approx 9 R$ and whose amplitude builds to a maximum amplitude of $\xi_{r 3}^{\text {obs }} / R \approx 0.1$ at an axial distance $\approx 18 R$ and declines in amplitude at larger axial distance; and (4) a rectangular distortion that rotates through a phase angle of $90^{\circ}$ over a wavelength $\lambda_{4} \approx 6 R$ as suggested by animation of the cross section images from the numerical simulation. The simulation animations indicate the presence of both $+n$ (clockwise-rotating) and $-n$ (counterclockwise-rotating) surface waves of equal amplitude that reinforce the square shape in $45^{\circ}$ increments with a multiply fluted crosssectional appearance at intermediate angles. The counterrotating waves reinforce in a linear additive fashion and grow to a maximum amplitude of $\xi_{r 4}^{\text {obs }} / R \approx 0.07 \quad(0.14$ combined) at an axial distance $\approx 18 R$ and decline in amplitude at larger axial distance.

The wavelengths used to fit the observed surface structures lie midway between the fastest growing wavelength and a precessionally induced wavelength for the helical mode, i.e., $12.5 R<\lambda_{1}<16.3 R$, and at a wavelength $50 \%$, $40 \%$, and $20 \%$ longer than the fastest growing wavelength for the elliptical, triangular, and rectangular modes, respec- 


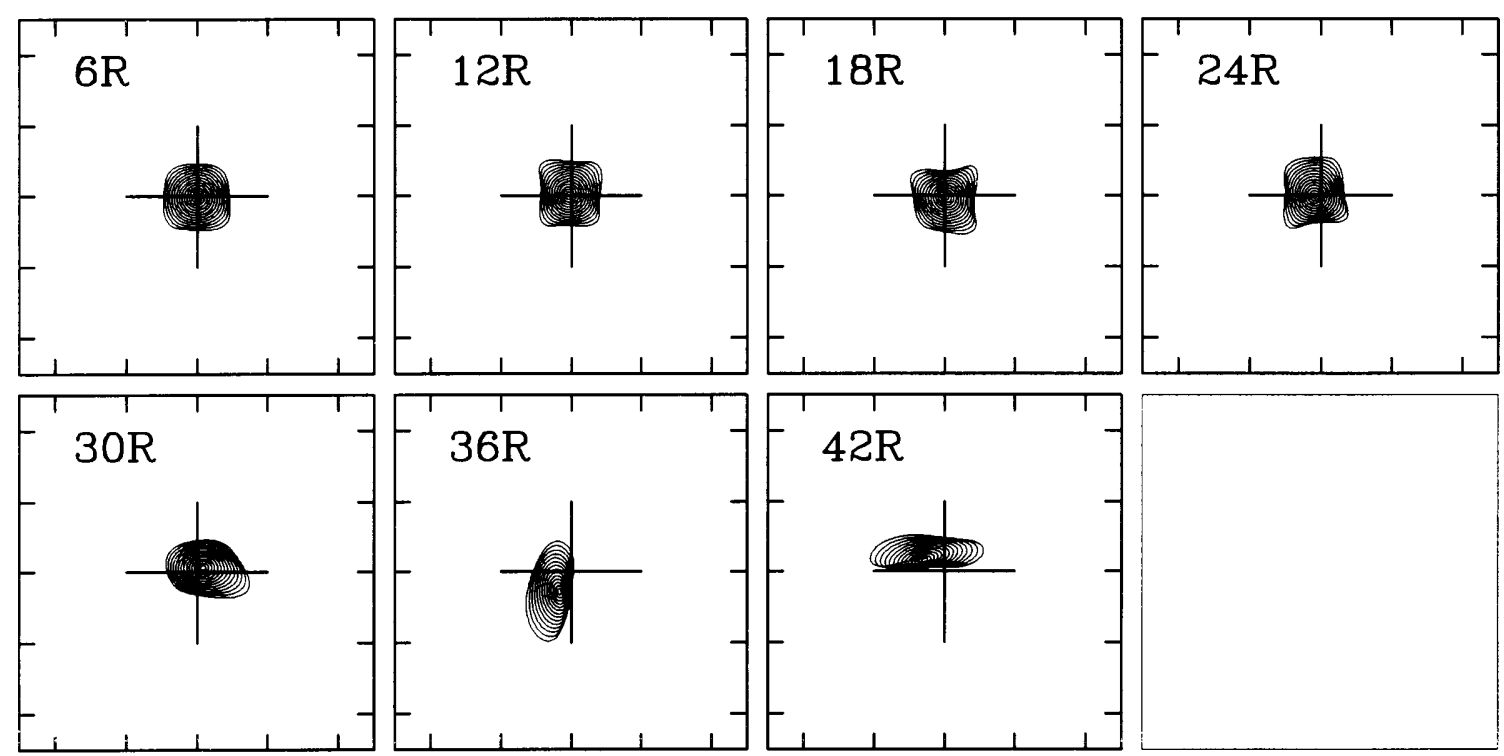

FIG. 14.-Fits to the surface distortions seen in the cross sections in Fig. 4 using the helical, elliptical, triangular, and rectangular surface modes only

tively. This result probably indicates a stronger coupling between the initial perturbation frequency and the helical mode and weaker coupling to the higher order modes. The surface displacements observed in the simulation have been fitted reasonably well using displacement surfaces computed from the linear theory even at the nonlinear amplitudes observed for the helical and elliptical distortions. The observed wavelength and the maximum observed surface displacements compared to the predicted maxima (computed from eqs. [8] and [10]) are shown in Table 3. The maximum observed surface displacements for helical and elliptical modes are approximately equal to the computed maxima. The maximum observed surface displacements for triangular and rectangular modes are less than the computed maxima. The transverse velocity perturbation as computed from equation (7b) required to accompany the observed displacements is less than the jet magnetosonic speed for all observed surface modes.

We have performed a preliminary investigation of the velocity structure of surface and body waves along the jet axis in an attempt to identify the periodicities observed in the velocity plots with either surface or body waves. In particular, let us concentrate on the velocity plots shown in Figure 8 for simulation C. Note that a multiplication by a factor 1.9 converts the velocity scale in units of $a_{\mathrm{ex}}$ in Figure 8 to multiples of the jet magnetosonic speed, $a_{\mathrm{jt}}^{\mathrm{ms}}$. Refer to Table 2 for the wavelengths of surface and body waves at maximum growth computed from the linear analysis. Interior to an axial distance of $30 R$, velocity fluctuation in $u_{x}$ ( $x$-velocity panel in Fig. 8) shows scale lengths, $1.5 R-$ $3.5 R$, that lie in the range of the first three pinch mode body waves. In general, in the linear limit, the higher order modes

TABLE 3

Observed Surface Mode Parameters in Simulation C

\begin{tabular}{ccccc}
\hline \hline Mode & $\lambda^{\text {obs }} / \lambda_{S}^{*}$ & $v_{w}^{\text {obs }} / v_{w, S}^{*}$ & $\xi_{r n}^{\text {obs }}$ & $\xi_{\text {pred }}^{\max }$ \\
\hline Helical $\ldots \ldots \ldots \ldots$ & 1.1 & $\sim 1.1$ & $\sim 0.8$ & $\sim 0.75$ \\
Elliptical $\ldots \ldots \ldots$. & 1.5 & $\sim 1.1$ & $\sim 0.6$ & $\sim 0.60$ \\
Triangular $\ldots \ldots .$. & 1.4 & $\sim 1.1$ & $\sim 0.1$ & $\sim 0.40$ \\
Rectangular $\ldots \ldots .$. & 1.2 & $\sim 1.1$ & $\sim 0.07$ & $\sim 0.25$ \\
\hline
\end{tabular}

can produce only relatively small velocity fluctuation in the axial direction. On the other hand, the large dips in $u_{x}$ at axial distances of $\sim 32 R$ and $\sim 48 R$ and accompanying transverse velocity maxima in $u_{y}$ and $u_{z}(y$ - and $z$-velocity panels in Fig. 8) are the result of a combination of large amplitude helical and elliptical jet perturbation. Basically the jet at these locations has been displaced off the axis (see, for example, Figs. 5 and 14) with axial velocity representative of the jet sheath.

The readily identifiable structures in the transverse velocity panels in Figure 8 are transverse velocity oscillation associated with the large-scale helical twist $(\lambda \approx 14 R)$, a relatively large transverse velocity oscillation at considerably smaller wavelength $(\lambda \approx 3 R)$ that can be identified with the first helical body wave, and still shorter wavelength $(\lambda \approx 1.5 R)$ transverse velocity oscillation structure in the ranges of the second and third helical body waves. We note that the transverse velocities observed along the jet axis, which can largely be attributed to the helical mode, are typically less than the jet magnetosonic speed (recall that $\left.a_{\mathrm{jt}}^{\mathrm{ms}} \sim 0.5 a_{\mathrm{ex}}\right)$ We note that the line-of-sight images showed dual twisted filaments internal to the jet $(\lambda \approx 4 R)$, which suggest the presence of internal elliptical body waves at axial distances $<30 R$. No significant transverse velocity structure along the jet axis is predicted to accompany this wave mode or with higher order wave modes. The observed body mode structures are not far above the limits imposed by the computational grid. It is likely that the amplitudes of these structures are influenced by the grid scale. A comprehensive investigation of internal jet structure and velocity awaits the completion and analysis of considerably higher resolution numerical simulations (Hardee et al. 1997).

\section{SUMMARY AND DISCUSSION}

A linearized analysis of supermagnetosonic jet stability predicts that the helical, elliptical, triangular, and rectangular surface wave modes can significantly influence jet dynamics and structure. Additionally, the first few body waves associated with pinch, helical, elliptical, triangular, and rectangular modes are also predicted to be capable of 
significantly influencing jet dynamics and structure. In the present simulations, the line-of-sight and cross section images show compelling evidence for helical, elliptical, triangular, and rectangular surface distortions and provide some evidence for pinch, helical, and elliptical body distortions. Helical and elliptical distortions are seen in the simulations at up to the maximum amplitudes predicted by the linear stability analysis. A rapid decline in the maximum amplitudes of waves for higher order modes is predicted by the linear analysis. However, a more rapid decline than predicted in the maximum amplitudes of the higher order surface wave modes is observed in the simulations; e.g., triangular and rectangular surface wave modes are seen at much less than the maximum amplitudes allowed by the linear theory. It is possible that the amplitudes of the observed higher order modes have been artificially reduced by the grid scale used in the simulations. Verification of this rapid decline in amplitudes for the higher order surface modes awaits completion and analysis of considerably higher resolution numerical simulations. Comparison between the present numerical simulations and a linear stability analysis shows that the linear analysis does a remarkable job of fitting jet structures even at the largest amplitudes observed.

Velocity structure in the numerical simulations has been compared to velocity structure predicted by the linear analysis to accompany surface wave modes. The velocity structure in the jet is consistent with the amplitudes of the surface distortions observed in the simulations. Velocity structure reveals the presence of jet body distortions that are not apparent in the line-of-sight images. In these simulations, the small transverse velocity supplied by precession at the jet origin, lack of jet rotation, and weak toroidal magnetic field provide a very small angular momentum. All wave modes other than the pinch mode consist of counterclockwise- (sense of jet precession and of magnetic helicity) and clockwise-growing components. The sense of jet precession and of magnetic helicity provide only a small bias toward the counterclockwise rotating components. In fact, nearly equal amplitude counterrotating components were observed for the rectangular mode. At a much lesser level, this effect may be present in the helical and elliptical modes but is obscured by the combined distortions.

All three jets show evidence for similar structure before helical and elliptical distortions grow to large amplitudes. Organized helical and elliptical structures persist at large amplitudes only for the jet denser than the external medium. For example, the jet less dense than the external medium loses structure and becomes plumelike as helical and elliptical distortions reach large amplitude and the jet entrains denser external material. Conversely, the dense jet maintains a high-speed spine surrounded by a low-speed sheath across the computational grid. A jet of density equal to that of the external medium is intermediate between these two cases and shows some evidence for a spine-sheath morphology, although the spine slows significantly on the computational grid. When compared to previous purely fluid fully three-dimensional simulations, our present simulation results show that the inclusion of a relatively strong, equipartition, nearly poloidal magnetic field does not significantly modify the linear structure or the nonlinear entrainment and slowing of a jet less dense than the external medium found by Hardee et al. (1995). Note also that the present simulations contain magnetic fields that are some- what stronger than might be expected in an astrophysical jet if a large lobe is to form (Clarke 1994) and contain no magnetic field in the external medium as might be expected within a lobe or cocoon formed from jet material. We do not expect reduction in the jet magnetic field or inclusion of a weak external magnetic field to change the present results substantially but additional simulations designed to study in more detail the effect of magnetic field orientation on structure, entrainment and mixing are in progress.

Although the magnetosonic Mach numbers in the three simulations are comparable, the jet velocity is $12.5 a_{\mathrm{ex}}, 5.14$ $a_{\mathrm{ex}}$, and $4.04 a_{\mathrm{ex}}$ in the light jet, equal density jet, and heavy jet simulations, respectively. Previous purely fluid light jet simulations showed that higher jet velocity leads to more rapid heating and disruption as jet and external fluids mix. Thus, in these simulations, it is likely that the rapid disruption of the light jet and lack of disruption of the heavy jet is at least partially attributable to the velocity difference and not just the density difference. However, note that the most stable combination (linearly and nonlinearly) for a given magnetosonic Mach number is always associated with the denser jet. This is so because for a fixed magnetosonic Mach number, which governs the spatial growth of perturbations in the linear regime, the denser jet will have a lower velocity relative to the external medium than the light jet and hence be less susceptible to nonlinear disruption.

The jets in extragalactic radio sources can extend to distances that are considerably larger in terms of the initial jet radius than the distances studied in these numerical simulations. Transverse perturbations to the jet may be in the form of the regular precession studied here, although at considerably different frequency, or may be more random in nature. That small transverse perturbations can propagate to very large distances has been demonstrated in twodimensional slab jet simulations. A slab jet is spatially resolved along two Cartesian axes and is effectively infinite in extent in the third dimension. A set of simulations of expanding "equilibrium" slab jets in suitable atmospheres has shown propagation and growth of sinusoidal oscillations out to distances over 200 times the initial jet radius (Hardee \& Clarke 1995). In these simulations, adiabatic jet expansion was shown to provide a predicted stabilizing influence through both increase in the local Mach number and increase in the fundamental length scale given by the local jet radius. More recent simulations of constant radius "equilibrium" slab jets in a constant atmosphere but with very high Mach numbers have shown propagation and growth of sinusoidal oscillations out to distances in excess of 600 times the jet radius (Stone, Xu, \& Hardee 1997). These slab jet simulations make it clear that small linear disturbances initiated near the origin of a jet can lead to consequences at much larger scales, and thus our present studies at relatively small spatial scales can be imagined to apply to much larger spatial scales.

Random transverse perturbations can be initiated at any point along a long jet, whereas periodic perturbations are almost certainly produced near the origin. Previous slab jet simulations have shown that a random perturbation excites wave mode development at the most unstable frequency and wavelength (Zhao et al. 1992). With no perturbation at the jet origin, a three-dimensional propagating jet simulation shows essentially no instability until random perturbations driven by asymmetric lobe turbulence excite wave mode development at the most unstable frequencies and 
wavelengths (Norman 1996). Previous numerical simulations of (slab) cylindrical jets undergoing periodic (oscillation) precession show that excitation of all wave modes other than the large-scale (sinusoid) helix occurs at or near the most unstable frequency and wavelength (cf. the higher order surface and body modes in these simulations). Periodic perturbations are not likely to be at the most unstable helical frequency as was the case in the present numerical simulations. However, it has been shown that low precession frequencies result in behavior like that seen in these simulations albeit with the longer helical wavelength and slower spatial growth predicted by the linear stability analysis; precession frequencies above the most unstable helical frequency should be capable of exciting only internal structures as the jet cannot respond bodily to higher frequencies (Hardee, Cooper, \& Clarke 1994; Stone et al. 1997). Since lower frequency precessional perturbations result in some stabilization as helical twisting is of longer wavelength and grows more slowly, somewhat slower development of the nonlinear behavior (primarily the result of helical and elliptical distortion) observed in these simulations is likely to be the most significant difference. Note that high-frequency precession may also lead to increased stability as large-scale helical twisting should be suppressed. This situation is sufficiently different from our present simulations that our present results should not be extended to the situation of rapid precession. Our results should also not be extended to the case of large amplitude nonlinear initial perturbations.

Magnetic jet acceleration and collimation schemes require and simple flux conservation arguments combined with jet expansion imply that magnetic fields in large-scale jets take on a primarily toroidal configuration different from the poloidal configuration studied here. Preliminary results indicate that weak toroidal and/or poloidal fields, i.e., plasma $\beta \equiv 8 \pi P_{\mathrm{th}} / B^{2} \gg 1$, will behave like the present simulations. Only strong toroidal magnetic fields will make a large difference in jet and radio source morphology. Note also that numerical simulations show that strong toroidal magnetic fields inhibit the formation of a large radio lobe (Clarke 1994), which suggests that strong toroidal fields are not present at large scales. Thus, we are confident that the present poloidally magnetized numerical simulations serve as a reliable indicator of the behavior of many extragalactic jet outflows on the largest scales.

The nonlinear behavior and appearance of the jets in these simulations suggests that FR I radio sources have a plumelike appearance, e.g., M84, or develop a plumelike appearance after exhibiting large-scale helical distortion, e.g., 3C 449 (see Hardee et al. 1994), as a result of jet interaction with a medium that is of comparable or greater density than the jet. This happens if the propagating jet does not form or cannot maintain a low-density lobe or cocoon that can protect the jet from a denser external environment. Note that formation and maintenance of a lobe or cocoon requires both a low-density jet with respect to the undisturbed external environment and sufficiently high pressure at the jet front to inflate a lobe and drive a backflow against the expected pressure gradients associated with a galactic atmosphere (see Hardee et al. 1992b). On the other hand, these simulations suggest that FR II radio sources can have long structured jets because the jet forms and maintains a lobe or cocoon of density much less than that of the jet through which the jet propagates without disruptive entrainment.

The present dense jet simulation shows that helical twisting and twisted elliptical jet distortion, which bifurcates the poloidal magnetic field into filaments, can exist over large distances without leading to rapid disruption of organized jet structures. Additionally, the elliptical distortion and accompanying magnetic filaments can twist at a rate much different from the helical twist in a dense jet (unlike the light jet). This jet morphology appears remarkably similar to the morphology of the Cygnus A jet (Carilli et al. 1996), which shows evidence for a filament pair that twists through $180^{\circ}$ over a distance comparable to that of the $360^{\circ}$ helical twist suggested by the sinusoidal oscillation of the jet in the plane of the sky, and polarization vectors suggesting primarily poloidal magnetic fields. Additionally, the dense jet simulation shows that a magnetic filament brightens when it is aligned with the leading edge of jet helicity, which leads to bright diagonal filaments that cross the jet with the same angle at regular intervals. Similar diagonal structure is also evident in the Cygnus A jet. Analysis of the structures in the Cygnus A jet indicates a jet with a relativistic Mach number $M_{\text {rel }} \equiv \gamma_{\text {rel }}\left(u / a_{\mathrm{jt}}\right) \approx 10$ (Hardee 1996), where $\gamma_{\text {rel }}$ is the Lorentz factor corresponding to the jet flow speed.

In the dense jet simulation, the jet as revealed by the total intensity image exhibits a relatively large amplitude transverse oscillation. This simulation shows that the jet consists of a small jet core embedded in a broader sheath. Thus, a modest transverse motion relative to the width of the core and sheath could appear as a relatively large displacement of the observed jet core. We note that the sheath in the present dense jet simulation extends somewhat beyond the region into which jet fluid and magnetic field have been mixed with the external medium. We cannot draw any firm conclusions regarding the transverse extent of a sheath in extragalactic jets from these simulations as numerical effects and the discrete grid lead to an effective Reynolds number different from that of an extragalactic jet. However, some of the qualitative aspects of our present simulations should hold true for extragalactic jets, and we should not be surprised if the highly collimated outflows seen in continuum radio images are embedded in more slowly moving outward-flowing material that may also exhibit helically twisted magnetized emission filaments at some distance from the jet core.

P. Hardee and A. Rosen acknowledge support from the National Science Foundation through grant AST 93-18397 to the University of Alabama. D. Clarke is supported in part by the Natural Sciences and Engineering Research Council of Canada (NSERC). The numerical work utilized the Cray C90 at the Pittsburgh Supercomputing Center through grant AST 93-0010P.

\section{APPENDIX}

Let us model a jet as a cylinder of radius $R$, having a uniform density, $\rho_{\mathrm{jt}}$, a uniform internal poloidal magnetic field, $B_{\mathrm{jt}}$, and a uniform velocity, $u$. The external medium is assumed to have a uniform density, $\rho_{\mathrm{ex}}$, and to contain no magnetic field. 
The jet is established in static total pressure balance with the external medium where the total static uniform pressure is $p_{\mathrm{jt}}^{*} \equiv p_{\mathrm{jt}}+B_{\mathrm{jt}}^{2} / 8 \pi=p_{\mathrm{ex}}^{*}=p_{\mathrm{ex}}$. The general approach to analyzing the stability properties of this system is to linearize the one-fluid MHD equations of continuity and momentum along with an equation of state within each medium where the flow velocity $u=0$. The flow velocity is then reintroduced when solutions are matched at the jet boundary. In each medium we assume that the perturbations are adiabatic in nature. The linearized ideal magnetohydrodynamic equations that are relevant to our model become

$$
\begin{gathered}
\frac{\partial \rho_{1}}{\partial t}+\nabla \cdot\left(\rho_{0} \boldsymbol{u}_{1}\right)=0, \\
\rho_{0} \frac{\partial \boldsymbol{u}_{1}}{\partial t}=-\nabla p_{1}+\frac{\left[\left(\boldsymbol{\nabla} \times \boldsymbol{B}_{0}\right) \times \boldsymbol{B}_{1}+\left(\boldsymbol{\nabla} \times \boldsymbol{B}_{1}\right) \times \boldsymbol{B}_{0}\right]}{4 \pi}, \\
\frac{\partial p_{1}}{\partial t}=-\Gamma p_{0}\left(\nabla \cdot \boldsymbol{u}_{1}\right),
\end{gathered}
$$

where the density, velocity, pressure, and magnetic field are written as $\rho=\rho_{0}+\rho_{1}, \boldsymbol{u}=\boldsymbol{u}_{1}, p=p_{0}+p_{1}, \boldsymbol{B}=$ $\boldsymbol{B}_{0}+\boldsymbol{B}_{1}$, and subscript 1 refers to a perturbation to the equilibrium quantity with subscript 0 .

In cylindrical geometry, we look for perturbations $\rho_{1}, \boldsymbol{u}_{1}, p_{1}$, and $\boldsymbol{B}_{1}$ of the form

$$
f_{1}(r, \phi, x)=f_{1}(r) \exp [i(k x \pm n \phi-\omega t)],
$$

where the flow is in the positive $x$-direction, and $r$ is in the radial direction with the flow bounded by $r=R$. With this form for the perturbations, the differential equation for the dependence of the total pressure perturbation $p_{1}^{*}=p_{1}+\left(\boldsymbol{B}_{1} \cdot \boldsymbol{B}_{0}\right) / 4 \pi$ within each fluid as a function of $r$ can be written in the form

$$
r^{2} \frac{\partial^{2}}{\partial r^{2}} p_{1}^{*}+r \frac{\partial}{\partial r} p_{1}^{*}+\left(\beta^{2} r^{2}-n^{2}\right) p_{1}^{*}=0
$$

where for poloidal magnetic fields

$$
\beta \equiv\left[-k^{2}+\frac{\omega^{4}}{\omega^{2}\left(a^{2}+V_{\mathrm{A}}^{2}\right)-k^{2} V_{\mathrm{A}}^{2} a^{2}}\right]^{1 / 2} .
$$

In equation (A3), $a=(\Gamma p / \rho)^{1 / 2}$ is the sound speed, and $V_{\mathrm{A}}=\left(B^{2} / 4 \pi \rho\right)^{1 / 2}$ is the Alfvén speed in the appropriate medium. The solutions that are well behaved at jet center and at infinity are

$$
p_{1 \mathrm{jt}}^{*}(r)=C_{\mathrm{jt}} J_{ \pm n}\left(\beta_{\mathrm{jt}} r\right), \quad \text { and } \quad p_{1 \mathrm{ex}}^{*}(r)=C_{\mathrm{ex}} H_{ \pm n}^{(1)}\left(\beta_{\mathrm{ex}} r\right)
$$

inside and outside the jet, respectively, where $J_{ \pm n}$ and $H_{ \pm n}^{(1)}$ are Bessel and Hankel functions. In equation (A4)

$$
\beta_{\mathrm{jt}}=\left[-k^{2}+\frac{(\omega-k u)^{4}}{(\omega-k u)^{2}\left(a_{\mathrm{jt}}^{2}+V_{\mathrm{A}, \mathrm{jt}}^{2}\right)-k^{2} V_{\mathrm{A}, \mathrm{jt}}^{2} a_{\mathrm{jt}}^{2}}\right]^{1 / 2}, \quad \text { and } \quad \beta_{\mathrm{ex}}=\left(-k^{2}+\frac{\omega^{2}}{a_{\mathrm{ex}}^{2}}\right)^{1 / 2}
$$

there is no magnetic field in the external medium, and $\omega \rightarrow \omega-k u$ has reintroduced the jet flow speed through the Dopplershifted frequency in $\beta_{\mathrm{j} t}$.

At $r=R$, we require that the total pressure be continuous across the boundary, i.e., $p_{1 \mathrm{jt}}^{*}(R)=p_{1 \mathrm{ex}}^{*}(R)$, and that the radial fluid displacement inside and outside the jet be equal at the jet boundary, i.e., $\xi_{r}^{\text {jt }}(R)=\xi_{r}^{\text {ex }}(R)$, where the fluid displacement in the radial direction, $\xi_{r}(r)$, is given by

$$
\xi_{r}^{\mathrm{jt}}(r)=\frac{1}{\chi_{\mathrm{jt}}} \frac{\partial p_{1 \mathrm{jt}}^{*}(r)}{\partial r}, \quad \text { and } \quad \xi_{r}^{\mathrm{ex}}(r)=\frac{1}{\chi_{\mathrm{ex}}} \frac{\partial p_{1 \mathrm{ex}}^{*}(r)}{\partial r}
$$

inside and outside the jet, respectively, and where

$$
\chi_{\mathrm{jt}}=\rho_{\mathrm{jt}}\left[(\omega-k u)^{2}-k^{2} V_{\mathrm{A}, \mathrm{jt}}^{2}\right], \quad \text { and } \quad \chi_{\mathrm{ex}}=\rho_{\mathrm{ex}} \omega^{2} .
$$

Equations (A4) and (A5) along with the boundary conditions on the pressure and radial displacement result in a dispersion relation

$$
\frac{\beta_{\mathrm{jt}}}{\chi_{\mathrm{jt}}} \frac{J_{n}^{\prime}\left(\beta_{\mathrm{jt}} R\right)}{J_{n}\left(\beta_{\mathrm{jt}} R\right)}=\frac{\beta_{\mathrm{ex}}}{\chi_{\mathrm{ex}}} \frac{H_{n}^{(1) \prime}\left(\beta_{\mathrm{ex}} R\right)}{H_{n}^{(1)}\left(\beta_{\mathrm{ex}} R\right)}
$$

describing the normal modes of a cylindrical jet. In equation (A6) the primes denote derivatives of the Bessel and Hankel functions with respect to their arguments. The $n=0,1,2,3$, 4, etc. modes involve pinching, helical, elliptical, triangular, rectangular, etc. distortions of the jet, respectively.

Inside the jet, the azimuthal and axial fluid displacements are related to the pressure perturbation by

$$
\xi_{\phi}^{\mathrm{jt}}(r)= \pm \frac{i}{\chi_{\mathrm{jt}}} \frac{n}{r} p_{1 \mathrm{jt}}^{*}(r), \quad \text { and } \quad \xi_{x}^{\mathrm{jt}}(r)=\frac{i}{\chi_{\mathrm{jt}}} k\left[\frac{\chi_{\mathrm{jt}}}{\chi_{\mathrm{jt}}+\left(B_{\mathrm{jt}}^{2} / 4 \pi\right)(\omega-k u)^{2} / a_{\mathrm{jt}}^{2}}\right] p_{1 \mathrm{jt}}^{*}(r)
$$


With the radial dependence of the total pressure perturbation written in the form

$$
p_{1 \mathrm{jt}}^{*}(r)=\frac{J_{n}\left(\beta_{\mathrm{jt}} r\right)}{J_{n}\left(\beta_{\mathrm{jt}} R\right)} p_{1 \mathrm{jt}}^{*}(R)
$$

the radial dependence of the radial, azimuthal, and axial displacement perturbations can all be written in terms of the radial displacement perturbation appropriate to wave mode $n$ at the jet surface:

$$
\begin{aligned}
& \xi_{r, n}^{\mathrm{jt}}(r)=\frac{J_{n}^{\prime}\left(\beta_{\mathrm{jt}} r\right)}{J_{n}^{\prime}\left(\beta_{\mathrm{jt}} R\right)} \xi_{r, n}^{\mathrm{jt}}(R), \\
& \xi_{\phi, n}^{\mathrm{jt}}(r)= \pm n \frac{i}{\beta_{\mathrm{jt}} r} \frac{J_{n}\left(\beta_{\mathrm{jt}} r\right)}{J_{n}^{\prime}\left(\beta_{\mathrm{jt}} R\right)} \xi_{r, n}^{\mathrm{jt}}(R), \\
& \xi_{x, n}^{\mathrm{jt}}(r)=k r\left[\frac{\chi_{\mathrm{jt}}}{\chi_{\mathrm{jt}}+\left(B_{\mathrm{jt}}^{2} / 4 \pi\right)(\omega-k u)^{2} / a_{\mathrm{jt}}^{2}}\right] \frac{i}{\beta_{\mathrm{jt}} r} \frac{J_{n}\left(\beta_{\mathrm{jt}} r\right)}{J_{n}^{\prime}\left(\beta_{\mathrm{jt}} R\right)} \xi_{r, n}^{\mathrm{jt}(R),}
\end{aligned}
$$

where we have used equation (A5) to write

$$
\xi_{r, n}^{\mathrm{jt}}(r)=\frac{J_{n}^{\prime}\left(\beta_{\mathrm{jt}} r\right)}{J_{n}^{\prime}\left(\beta_{\mathrm{jt}} R\right)} \xi_{r, n}^{\mathrm{jt}}(R)=\frac{\beta_{\mathrm{jt}}}{\chi_{\mathrm{jt}}} \frac{J_{n}^{\prime}\left(\beta_{\mathrm{jt}} r\right)}{J_{n}\left(\beta_{\mathrm{jt}} R\right)} p_{1 \mathrm{jt}}^{*}(R) .
$$

Displacements of jet fluid from an initial position $(r, \phi, x)$ are given by $\xi(r, \phi, x)=\xi(r) \exp [i(k x \pm n \phi-\omega t)]$ with the $\xi(r)$ given by equations (A9), and $\beta_{\mathrm{jt}}=\beta_{\mathrm{jt}}(\omega, k)$ and $\chi_{\mathrm{jt}}=\chi_{\mathrm{jt}}(\omega, k)$ where $\omega$ and $k$ are normal mode solutions of the dispersion relation (A6). The velocity components associated with these displacements are given by $\boldsymbol{u}_{1}(r, \phi, x)=d \xi / d t=-i(\omega-k u) \xi(r$, $\phi, x)$. In general, $\xi(r)$ is complex and $\xi(r, \phi, x)$ can be written in the form $\xi\left(r, \phi_{s}, x_{s}\right)=\boldsymbol{A}(r) e^{i(r)} \xi_{r, n}^{\mathrm{jt}}(R) \exp \left[i\left(k x_{s} \pm n \phi_{s}-\omega t\right)\right]$ where $\phi_{s}$ and $x_{s}$ are now the azimuthal angle and axial position at the jet surface, and

$$
\begin{aligned}
A_{r}(r) e^{i \Delta_{r}(r)} & \equiv \frac{J_{n}^{\prime}\left(\beta_{\mathrm{jt}} r\right)}{J_{n}^{\prime}\left(\beta_{\mathrm{jt}} R\right)} \\
A_{\phi}(r) e^{i\left(\Delta_{\phi}(r) \pm \pi / 2\right)} & \equiv n \frac{1}{\beta_{\mathrm{jt}} r} \frac{J_{n}\left(\beta_{\mathrm{jt}} r\right)}{J_{n}^{\prime}\left(\beta_{\mathrm{jt}} R\right)} e^{ \pm i \pi / 2} \\
A_{x}(r) e^{i\left(\Delta_{x}(r)+\pi / 2\right)} & \equiv k r\left[\frac{1}{\chi_{\mathrm{jt}}+\left(B_{\mathrm{jt}}^{2} / 4 \pi\right)(\omega-k u)^{2} / a_{\mathrm{jt}}^{2}}\right] \frac{1}{\beta_{\mathrm{jt}} r} \frac{J_{n}\left(\beta_{\mathrm{jt}} r\right)}{J_{n}^{\prime}\left(\beta_{\mathrm{jt}} R\right)} e^{i \pi / 2},
\end{aligned}
$$

where we have set $\pm i=e^{ \pm i \pi / 2}$. Thus, we see that displacements are modified in amplitude by a factor $A(r)$ relative to those at the jet surface and rotated in azimuthal angle or shifted along the jet axis by $\Delta(r)$.

To make a transformation to Cartesian coordinates such as are used in the numerical simulations, we will set $\exp (i \theta)=\cos \theta$ with $\cos \theta=1$ along the $z$-axis, where the flow is along the $x$-axis. In this case, a point at $\left(x_{0}=x_{s}\right.$, $\left.y_{0}=-r \sin \phi_{s}, z_{0}=r \cos \phi_{s}\right)$ is displaced $(\delta x, \delta y, \delta z)$ by

$$
\begin{gathered}
\delta x=A_{x}(r) \xi_{r, n}^{\mathrm{jt}}(R) \cos \left[k x_{s}-\omega t \pm n \phi_{s}+\Delta_{x}(r)+\pi / 2\right], \\
\delta y=-A_{r}(r) \xi_{r, n}^{\mathrm{jt}}(R) \cos \left[k x_{s}-\omega t \pm n \phi_{s}+\Delta_{r}(r)\right] \sin \phi_{s}-A_{\phi}(r) \xi_{r, n}^{\mathrm{jt}}(R) \cos \left[k x_{s}-\omega t \pm n \phi_{s}+\Delta_{\phi}(r) \pm \pi / 2\right] \cos \phi_{s}, \\
\delta z=A_{r}(r) \xi_{r, n}^{\mathrm{jt}}(R) \cos \left[k x_{s}-\omega t \pm n \phi_{s}+\Delta_{r}(r)\right] \cos \phi_{s}-A_{\phi}(r) \xi_{r, n}^{\mathrm{jt}}(R) \cos \left[k x_{s}-\omega t \pm n \phi_{s}+\Delta_{\phi}(r) \pm \pi / 2\right] \cos \phi_{s} .
\end{gathered}
$$

Of particular interest are distortions to the initially circular jet cross section. In the numerical simulation, a cross section is obtained at a fixed axial distance and time, say at $x_{s}=0$ and $t=0$, and the cross section of a displacement surface can therefore be constructed by obtaining $\delta x\left(r, \phi_{s}, x_{s}\right)=\delta x\left(r, \phi_{s}, 0\right)$ from equation (A11a) and then by obtaining $y_{0}+\delta y$ and $z_{0}+\delta z$ with $x_{s} \rightarrow-\delta x\left(r, \phi_{s}\right)$ from equations (A11b) and (A11c). This axial shift invokes a small additional azimuthal angle or axial shift of $-k_{R} \delta x\left(r, \phi_{s}\right)$ in addition to that arising from $\Delta(r)$. If displacements associated with many different surface and body wave modes are assumed to add in a linear fashion but each mode is oriented differently in the $y-z$ plane, then we may set $\phi_{s}=\phi_{s}+\Delta_{n m}$ in equations (A11a), (A11b), and (A11c), where $\Delta_{n m}$ provides the azimuthal or axial shift relative to the $z$-axis or $x$-axis, respectively, for the appropriate surface or body wave mode. In Cartesian coordinates, the total velocity will be given by $\boldsymbol{u}(x, y, z)=\boldsymbol{u}_{0}+\boldsymbol{u}_{1}(x, y, z)=\boldsymbol{u}_{0}-i(\omega-k u) \boldsymbol{\delta}\left(r, \phi_{s}, x_{s}\right)$, where $\boldsymbol{u}_{0}$ is the jet velocity along the $x$-axis, the components of $\delta\left(r, \phi_{s}, x_{s}\right)$ are given by equations (A11a), (A11b), and (A11c), and $(x, y, z)=\left(x_{s}+\delta x, y_{0}+\delta y, z_{0}+\delta z\right)$.

\section{REFERENCES}

Appl, S., \& Camenzind, M. 1992, A\&A, 256, 354 Bicknell, G. V. 1984, ApJ, 286, 68

.1986a, ApJ, 300, 591 .1986b, ApJ, 305, 109

Bodo, G., Massaglia, S., Rossi, P., Rosner, R., Malagoli, A., \& Ferrari, A. 1995, Ä\&A, 303, 281

Bodo, G., Rosner, R., Ferrari, A., \& Knoblock, E. 1989, MNRAS, 341, 631

Burke, T., Mundt, R., \& Ray, T. P. 1988, A\&A, 200, 99
Carilli, C. L., Perley, R. A., Bartel, N., \& Dreher, J. W. 1996, in Cygnus A-Study of a Radio Galaxy, ed. C. L. Carilli \& D. E. Harris (Cambridge: Cambridge Univ. Press), 76

Clarke, D. A. 1994, in Jets in Extragalactic Radio Sources, ed. H.-J. Röser \& K. Meisenheimer (Heidelberg: Springer), 243 . 1996, ApJ, 457, 291

Clarke, D. A., Norman, M. L., \& Burns, J. O. 1989, ApJ, 342, 700

Dreher, J. W., Carilli, C. L., \& Perley, R. A. 1987, ApJ, 316, 611 
Ferrari, A., Trussoni, E., \& Zaninetti, L. 1981, MNRAS, 196, 1051

Fiedler, R., \& Jones, T. W. 1984, ApJ, 283, 532

Hardee, P. E. 1983, ApJ, 269,94 1995, Ann. NY Acad. Sci., 773, 14

. 1996, in Cygnus A-Study of a Radio Galaxy, ed. C. L. Carilli \& D. E. Harris (Cambridge: Cambridge Univ. Press), 113

Hardee, P. E., \& Clarke, D. A. 1995, ApJ, 451, L25

Hardee, P. E., Clarke, D. A., \& Howell, D. A. 1995, ApJ, 441, 644

Hardee, P. E., Cooper, M. L., \& Clarke, D. A. 1994, ApJ, 424, 126

Hardee, P. E., Cooper, M. L., Norman, M. L., \& Stone, J. M. 1992a, ApJ, 399,478

Hardee, P. E., White, R. E., III, Norman, M. L., Cooper, M. A., \& Clarke, D. A. 1992 b, ApJ, 387, 460

Hardee, P. E., et al. 1997, in preparation

Hjellming, R. M., \& Rupen, M. P. 1995, Nature, 375, 464

Laing, R. A., \& Bridle, A. H. 1987, MNRAS, 228, 557

Mirabel, I. F., \& Rodríguez, L. F. 1994, Nature, 371, 46

Norman, M. L. 1996, in ASP Conf. Proc. 100, Energy Transport in Radio Galaxies and Quasars, ed. P. E. Hardee, A. H. Bridle \& J. A. Zensus (San Francisco: ASP), 319
Norman, M. L., Winkler, K.-H. A., \& Smarr, L. L. 1984, in Physics of Energy Transport in Extragalactic Radio Sources, ed. A. H. Bridle \& J. A. Eilek (Green Bank: NRAO), 150

Perley, R. A., Bridle, A. H., \& Willis, A. G. 1984, ApJS, 54, 291

Perley, R. A., \& Cornwell, T. J. 1984, in Physics of Energy Transport in Extragalactic Radio Sources, ed. A. H. Bridle \& J. A. Eilek (Green Bank: NRAO), 39

Ray, T. P. 1981, MNRAS, 196, 195

Rosen, A., Hardee, P. E., Clarke, D. A., \& Johnson, A. 1997a, in ASP Conf. Proc. 123, Proc. of the 12th Kingston Meeting on Theoretical Astrophysics: Computational Astrophysics, ed. D. A. Clarke \& M. J. West (San Francisco: ASP), 262

Rosen, A., et al. 1997b, in preparation

Stone, J. M., Hawley, J. F., Evans, C. E., \& Norman, M. L. 1992, ApJ, 388, 19

Stone, J. M., Xu, J., \& Hardee, P. E. 1997, ApJ, 483, 136

Tingay, S. J., et al. 1995, Nature, 374, 141

van Leer, B. 1977, J. Comput. Phys., 23, 276

Zhao, J.-H., Burns, J. O., Norman, M. L., \& Sulkanen, M. E. 1992, ApJ, 387,83 

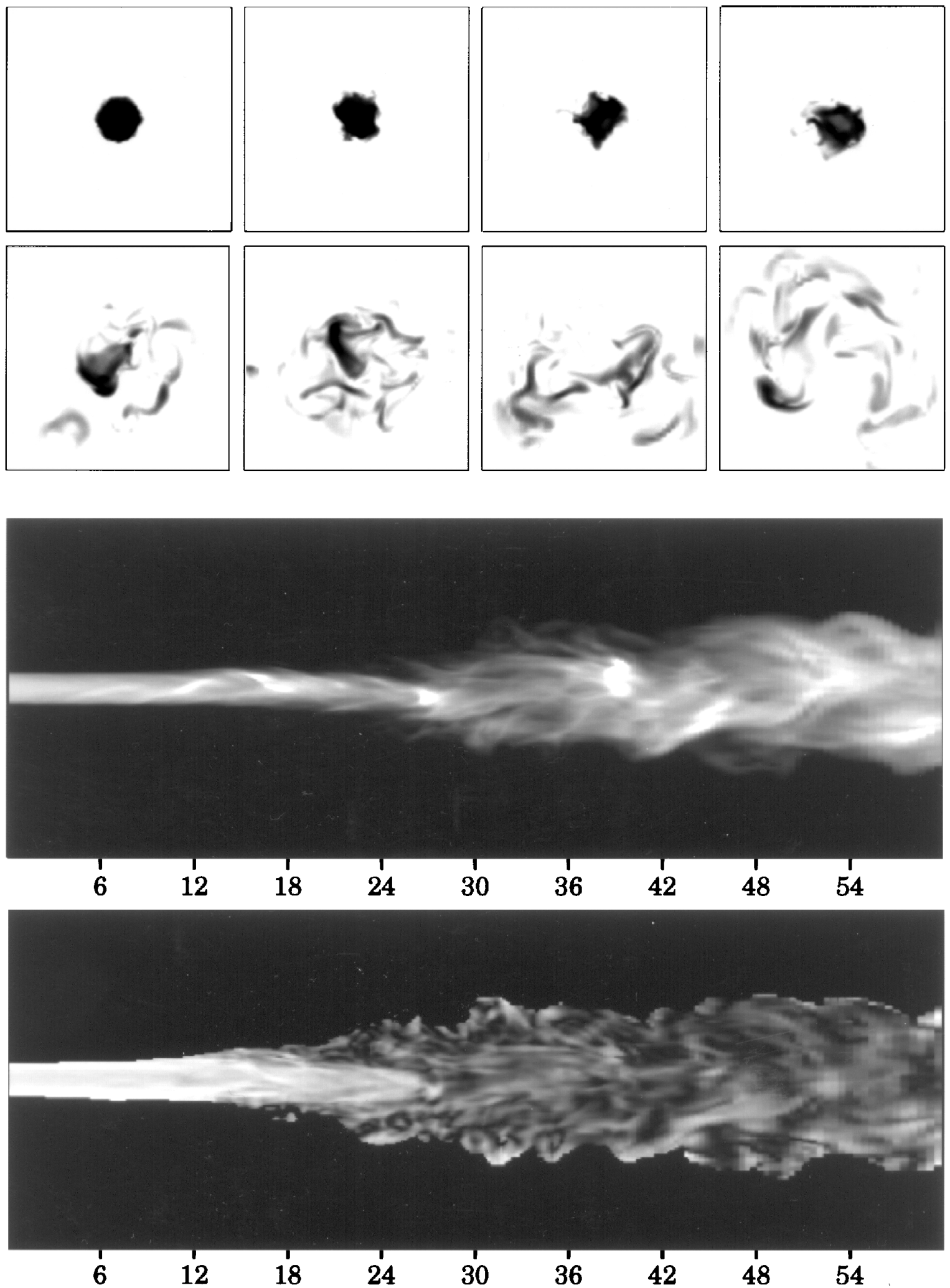

Fig. 2.-Magnetic pressure cross section images from simulation $A$ at axial distances from $6 R$ to $48 R$ in $6 R$ increments from top left to bottom right. The $y$-axis is in the vertical direction, the $z$-axis (line-of-sight axis) is in the horizontal direction, and the flow direction ( $x$-axis) is into the page. Each cross section image has dimension $10 R \times 10 R$ and darker indicates higher values of the magnetic pressure. Below the cross section images are two images of dimension $20 R \times 60 R$ showing (top) total intensity and (bottom) fractional polarization where the brightest regions are nearly $70 \%$ polarized. Markings at $6 R$ intervals show where cross sections and transverse velocity profiles are located.

HARDEE et al. (see 485, 535) 

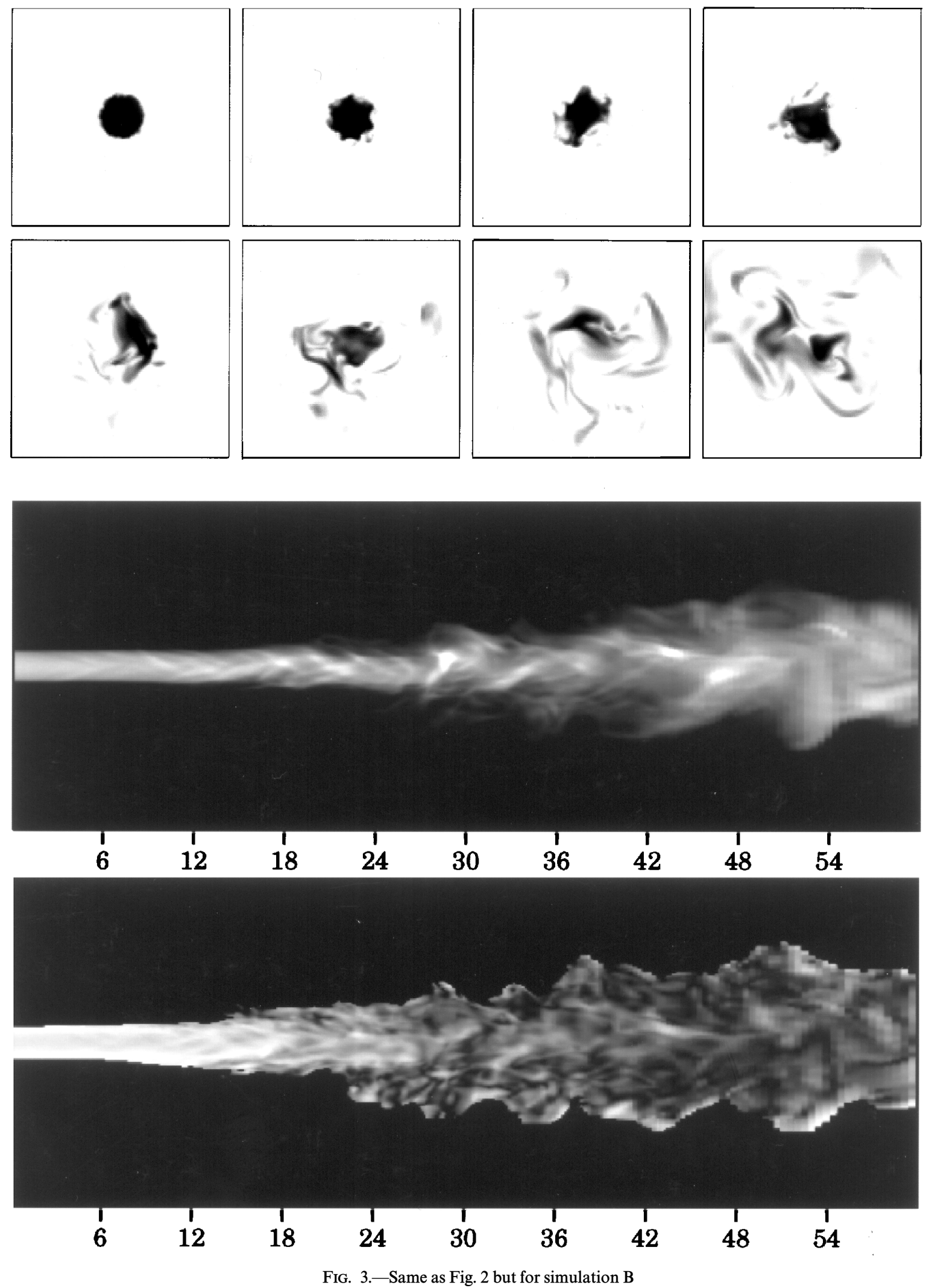

HARDEE et al. (see 485, 535) 

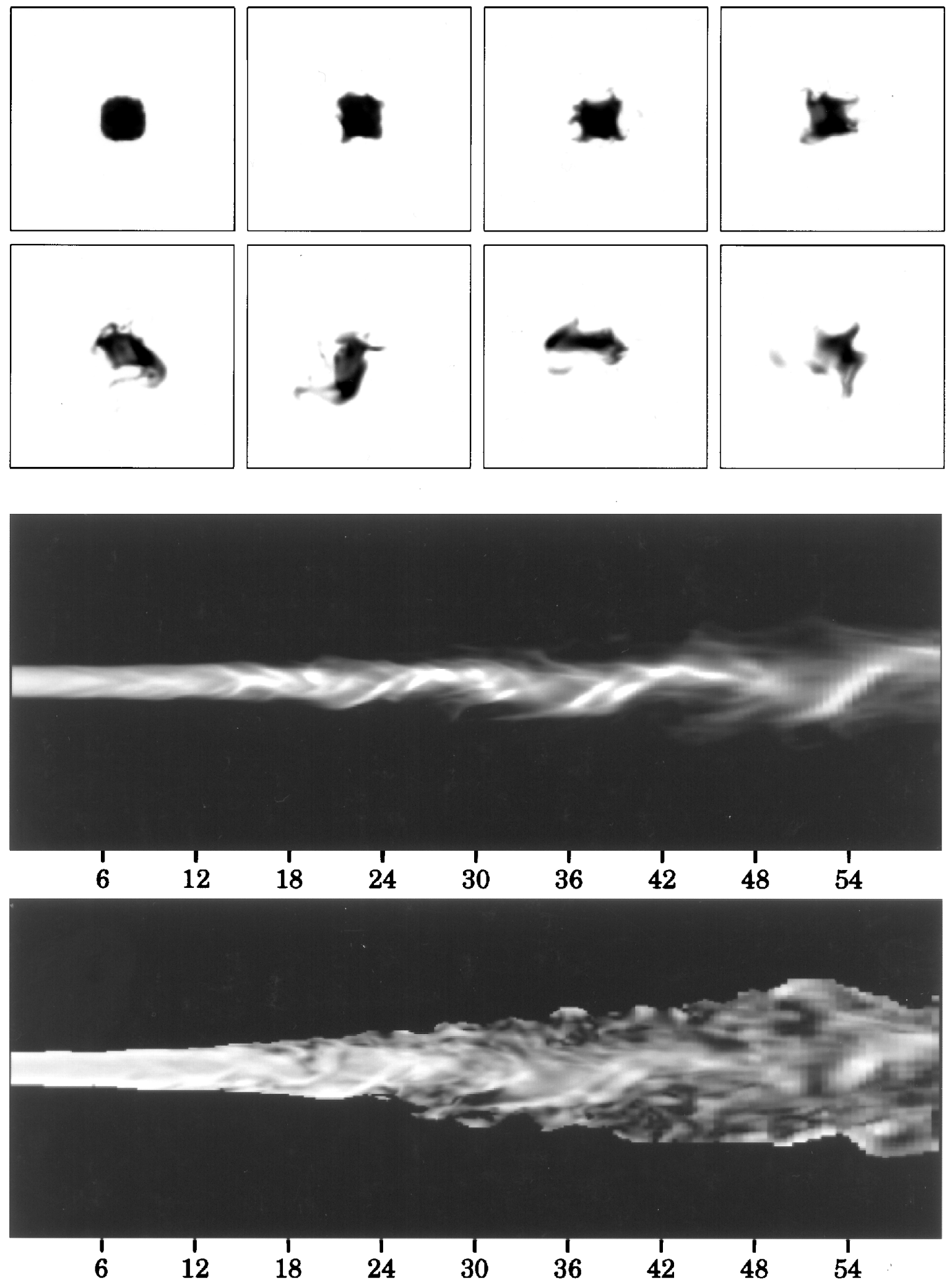

Fig. 4.-Same as Fig. 2 but for simulation $\mathrm{C}$ at time $\tau_{d}=34$

HARDEE et al. (see 485, 535) 

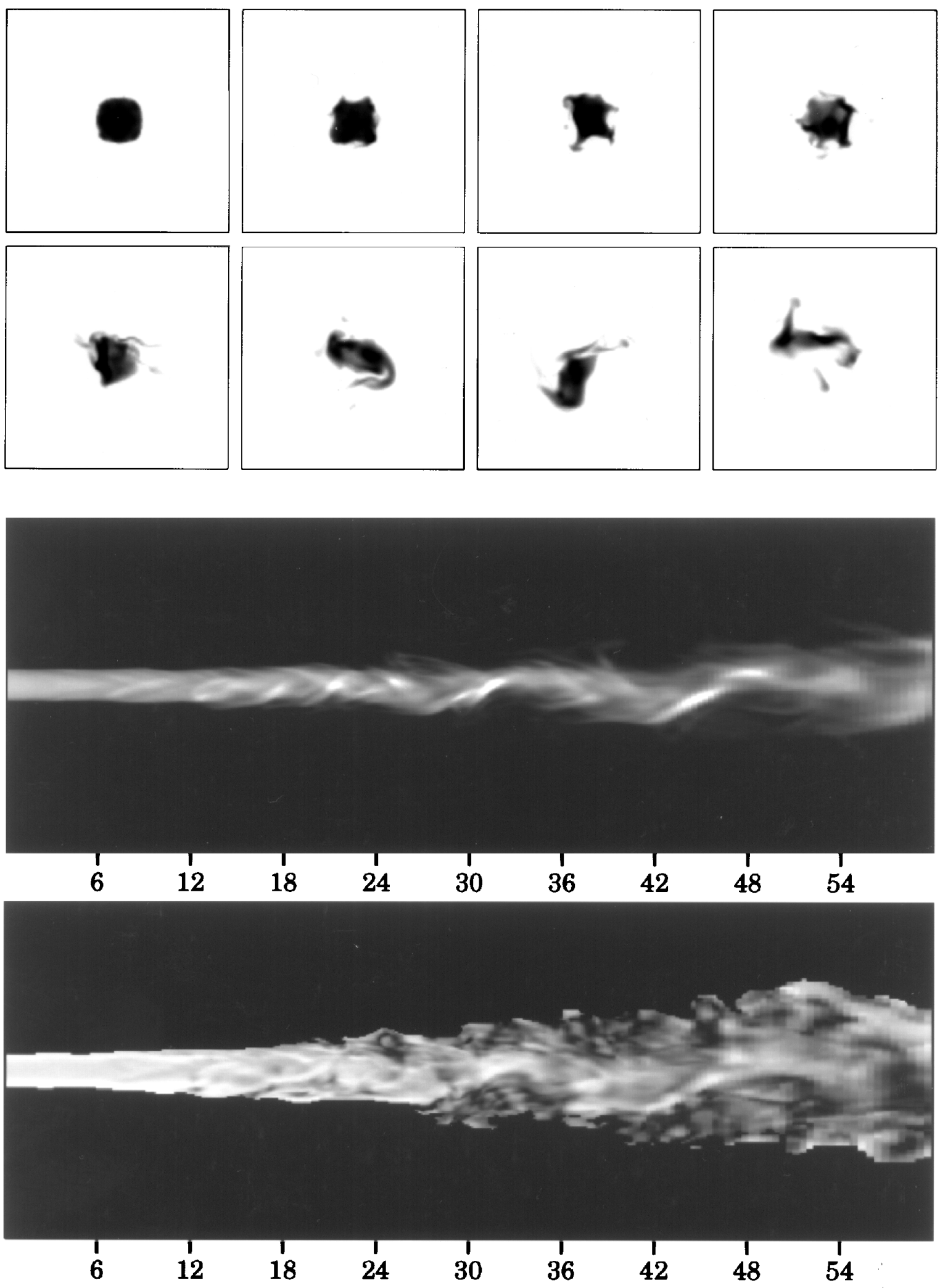

FIG. 5.-Same as Fig. 2 but for simulation C at time $\tau_{d}=36$

HARDEE et al. (see 485, 535) 